Article

\title{
A Fast and Convenient Synthesis of New Water-Soluble, Polyanionic Dendrimers
}

\author{
Grzegorz M. Salamończyk
}

check for updates

Citation: Salamończyk, G.M. A Fast and Convenient Synthesis of New Water-Soluble, Polyanionic Dendrimers. Molecules 2021, 26, 4754. https://doi.org/10.3390/

molecules26164754

Academic Editor: György Keglevich

Received: 22 July 2021

Accepted: 3 August 2021

Published: 6 August 2021

Publisher's Note: MDPI stays neutral with regard to jurisdictional claims in published maps and institutional affiliations.

Copyright: (C) 2021 by the author. Licensee MDPI, Basel, Switzerland. This article is an open access article distributed under the terms and conditions of the Creative Commons Attribution (CC BY) license (https:/ / creativecommons.org/licenses/by/ $4.0 /)$.
Centre of Molecular and Macromolecular Studies, The Polish Academy of Sciences, Sienkiewicza 112, 90-363 Łódź, Poland; gmsalamo@cbmm.lodz.pl; Tel.: +48-42-684-7113; Fax: +48-42-684-7126

\begin{abstract}
Reasonably simple, efficient, and possessing aspects of generality, the methodology for the synthesis of new, water-soluble, dendrimeric polyesters with great potential applications as antiviral drugs in their own right is described. The essential aspect of the presented approach is a quite unique, immediate access to the polyanionic material at each generation during divergent synthesis. Six target polyanionic dendrimers (generations 1, 2, and 3) have been synthesized. The key monomers applied in this project were 1,3,5-benzenetricarboxylic acid derivatives, which also worked as direct precursors of the charged dendrimer surface.
\end{abstract}

Keywords: dendrimer; polyanion; 1,3,5-benzenetricarboxylic acid; polyester; phosphorus; total synthesis; chemoselectivity

\section{Introduction}

Dendrimers are three-dimensional, highly branched, synthetic polymers that possess a well-defined structure. Their graphical representation resembles tree-like units emanating from a common spot. Naturally, dendrimers have been extensively studied for a variety of biomedical applications involving both therapy and diagnosis. However, this research seems to be still at an early stage [1-3]. Normally, antiviral and other drug research concentrates on relatively low molecular weight structures. On the contrary, dendrimers with their structural precision and with a large number of functional groups on the surface may serve as antiviral drugs in their own right [4-6]. Different types and generations of dendrimers substituted with different charged groups have been explored in the prevention and treatment of HIV. These macromolecular drug candidates can be administered as topical microbicides [7-9]. Topical microbicides interfere with the virus at the early stage of virus infection sequence, which is adsorption and fusion of the virus to the cell. The common feature of all such microbicide compounds is the presence of polyanions, which have the capability to bind to the (e.g., the so-called gp120 protein or spike protein (in the case of SARS-CoV-2)) outer protein located on the viral surface, thus inhibiting the entire viral infection process [10]. There are numerous examples of this type of compounds that can be found in the literature [11-14]. Thanks to their mode of action, polyanionic dendrimers can be expected to be effective against a broad spectrum of pathogenic viruses. In contrast to polycationic compounds, polyanionic dendrimers usually possess much lower cytotoxicity $[15,16]$. Definitely, the most successful polyanionic dendrimer used for antiviral purposes is SPL7013 (also known as VivaGel ${ }^{\circledR}$ or VIRALEZETM_Starpharma Ltd. A company developing dendrimer products for pharmaceutical applications, Australia), a sulfonated (32 terminal sulfonate groups) polylysine dendrimer (molec. weight $16.6 \mathrm{kD}$ ) [17]. VivaGel ${ }^{\circledR}$ was originally developed as a topical microbicide for the avoidance of sexually transmitted infections (STIs) such as HIV, HSV-2 (genital herpes), and human papillomavirus (HPV). There is an enormous global need for a vaginal microbicide offering protection of this nature. It has also been licensed as an antiviral condom coating. This compound went effectively through all the stages of clinical trials [18]. Except STIs causing viruses, the SPL7013 dendrimer possesses significant activity against 
other pathogenic viruses such as adenovirus, HBV (hepatitis B), RSV (respiratory syncytial virus) or Zika virus. Moreover, in April 2020, it was declared that the same dendrimer SPL 7013 showed significant antiviral activity against the coronavirus (SARS-CoV-2) that causes COVID-19 (coronavirus disease) [19]. Later on, in September 2020, Starpharma announced that SPL 7013 when applied at clinically relevant concentrations as a nasal spray (VIRALEZE ${ }^{\mathrm{TM}}$ ), had potent virucidal activity, inactivating more than $99.9 \%$ of highly infectious SARS-CoV-2.9 [20]. Therefore, VIRALEZE ${ }^{\mathrm{TM}}$ can be complementary to vaccines and other preventive measures.

Consequently, the chemical synthesis of potential antiviral compounds, including macromolecular material, such as water-soluble, charged dendrimers, possessing various types of tunable scaffolds and surfaces, is extremely important and very much justified.

\section{Results and Discussion}

Several years ago, we developed a method for the synthesis of dendrimeric polyphosphates and their analogs [21-25]. Later on, also from our laboratory, an effective syntheses of new polyester dendrimers based on a trimesic acid framework derivative have been disclosed $[26,27]$. In this research paper, a general and undemanding approach to the synthesis of carboxylate-terminated (polyanionic) dendrimers is reported. The key feature of this approach is the unprecedented formation and straightforward access to a polyanionic dendrimer at each generation during the divergent synthesis. Therefore, there is no need for often complicated, post-synthetic surface modification, and at least one difficult synthetic step is saved. Recently, we have found that medium-size (4.2-4.7 kD) carboxylateterminated polyanionic dendrimers [28] displayed exceedingly strong antiviral activity against both strains of HIV, way below their cytotoxicity versus MT4 cells [29]. Therefore, for a powerful antiviral activity, the high generation structures are not necessarily essential.

\subsection{Synthesis of Monomers}

The presented synthetic work commences from the preparation of both interior and surface unit monomers. The careful reaction of commercially available 1,3,5benzenetricarbonyl trichloride with 2.0 equiv. of lithium tert-butoxide, followed by a mild basic hydrolysis of the remaining acid chloride, produced chemoselectively the corresponding 1,3,5-benzenetricarboxylic acid di-tert-butyl ester or di-tert-butyl trimesoate (1) $(48 \%)$.

(Accompanied with the corresponding triester $(11 \%)$ and monoester $(17 \%)$, acc. to NMR) in $48 \%$ isolated yield. (Scheme 1) The synthesis (very low yield-22\% in a twostep procedure) of diester $\mathbf{1}$ has been recently reported [30]. Another highly chemoselective reaction was a reduction ( $\mathrm{rt}, 24 \mathrm{~h}$ ) of one carboxyl group in diester 1 using the borane-dimethyl sulfide complex, which provided the key reagent-di-tert-butyl 5-hydroxymethylbenzene-1,3-dicarboxylate (2) in a high isolated yield (86\% after crystallization from $\mathrm{CH}_{2} \mathrm{Cl}_{2}$-cyclohexane). This compound (2) represents an $\mathrm{AB}_{2}$-type monomer. The A group (hydroxyl) is active and the $\mathrm{B}$ groups (carboxyl) are protected such that the A group reacts solely with the $\mathrm{B}$ (active) groups in the prior generation of the dendrimer. Deprotection is necessary to activate the $B$ groups for the consequent reaction. This deprotection may not be quantitative and may also include undesired reactions, causing imperfections in the dendrimer skeleton. Therefore, the protective groups applied in this work were selected very cautiously. It will be demonstrated that deprotection reactions (cleavage of tert-butyl esters in the presence of benzoate-type esters) were complete and did not cause any defects in the expected structures. 


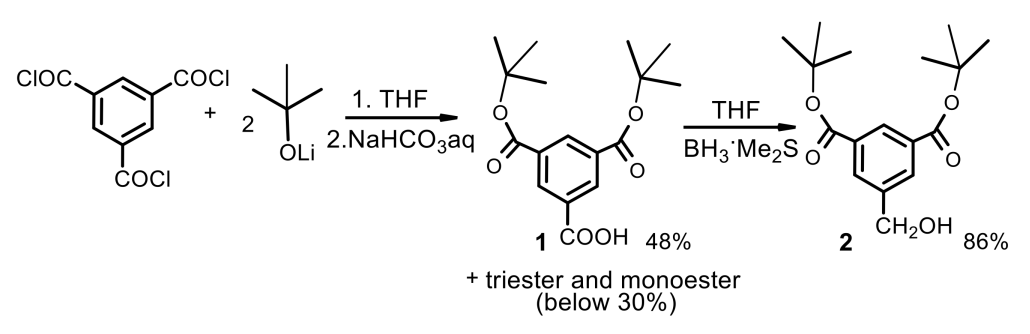

Scheme 1. Synthesis of the monomers 1 and 2.

\subsection{Synthesis of Dendrimers}

To express the usefulness of the presented synthetic methodology, the author split this project into three subsections.

Firstly, the core compound, tris(4-hydroxybutyl) phosphate [28] (3) was allowed to react with an excess of dibenzyl 1,3,5-benzenetricarboxylate [28], (1a) in the presence of the water-soluble carbodiimide, 1-ethyl-3-(3-dimethylaminopropyl)carbodiimide (EDC) and 4-dimethylaminopyridine (DMAP) in anhydrous dichloromethane to furnish the first generation (G1) dendrimer 4 in $92 \%$ isolated yield. (Scheme 2) The cleavage of the terminal benzyl esters in 4 using catalytic hydrogenolysis proceeded smoothly and quantitatively as evidenced by the ${ }^{1} \mathrm{H}$ NMR to provide pure acid 5 (no trace of protons corresponding to benzyl groups in 5 was detected) (96\%), which can be effortlessly transformed into the corresponding hexaanion 6 using, e.g., aqueous sodium bicarbonate. Then, hexacarboxylic acid 5 reacted readily with an excess of alcohol 2 using EDC and DMAP as condensation reagents (in $\mathrm{CH}_{2} \mathrm{Cl}_{2}$-THF 1:1 solution) to afford the second generation (G2) dendrimer 7 in $88 \%$ isolated yield.

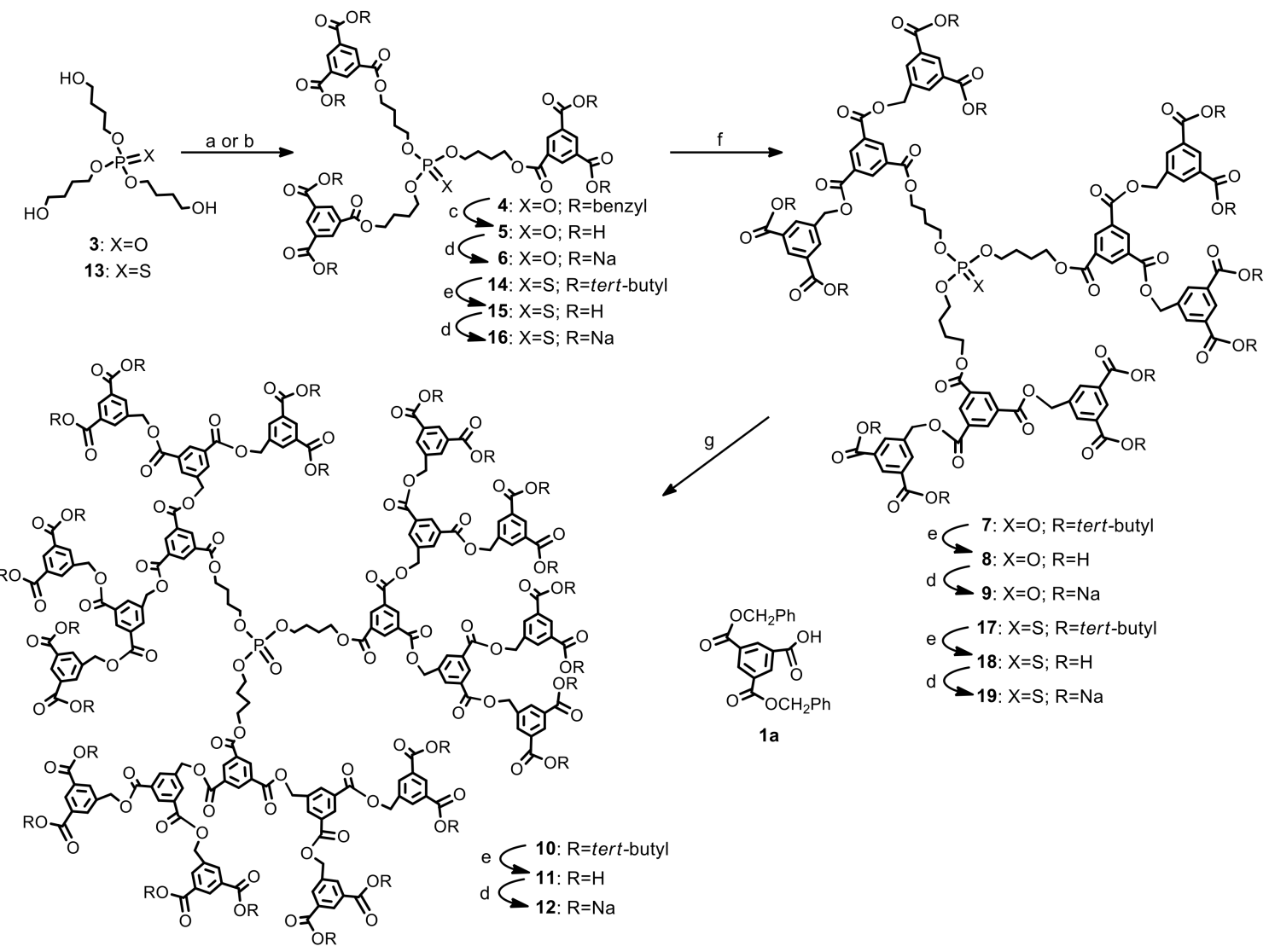

Scheme 2. Reagents and conditions: (a) for 3 as the substrate: acid 1a, EDC, DMAP, DCM, rt; (b) for 13 as the substrate: acid 1, EDC, DMAP, DCM, rt; (c) $\mathrm{H}_{2}$, Pd/C, $\mathrm{MeOH}$; (d) $\mathrm{NaHCO}_{3}$ aq.; (e) $\mathrm{CF}_{3} \mathrm{COOH}, \mathrm{DCM}$, rt; (f) alcohol 2, EDC, DMAP, DCM, THF, rt; (g) for dodecaacid 8 as the substrate: alcohol 2, EDC, DMAP, DCM, DMF, rt. 
Next, polyester $\mathbf{7}$ was stirred in a trifluoroacetic acid-dichloromethane 3:2 mixture at room temperature. The reaction was closely monitored by the ${ }^{1} \mathrm{H} N M R$. After $4 \mathrm{~h}$, neither the trace of protons related to tert-butyl residues nor any damage (cleavage of an internal ester bond) to the dendrimer skeleton was detected. The expected polyacid 8 was obtained in $90 \%$ isolated yield (all of the polyacids $(5,8, \mathbf{1 1}, \mathbf{1 5}, \mathbf{1 8}, \mathbf{2 2})$ reported here were purified by their precipitation from a THF solution with acetone). At this stage, another polyanionic (dodecaanion) dendrimer 9 (sodium salt of 8) was in hand. Repetition of the condensation of acid 8, this time, with alcohol 2 provided the third generation polyester $\mathbf{1 0}$ with a $76 \%$ isolated yield. It should be noted that the condensation of polyacid 8 with alcohol 2 requires the DCM-DMF mixture as the solvent due to its (acid 8) solubility issues. The structures, monodispersity, and high purity of all the dendrimeric products were confirmed by the NMR and MALDI TOF mass spectrometry. For instance, Figure 1 shows the MALDI TOF mass spectrum of third generation dendrimer 10. The signals at 5464.7 and 5477.8 are attributed to the molecular ion $(\mathrm{M}+\mathrm{Na})$ and $(\mathrm{M}+\mathrm{K})$, respectively.

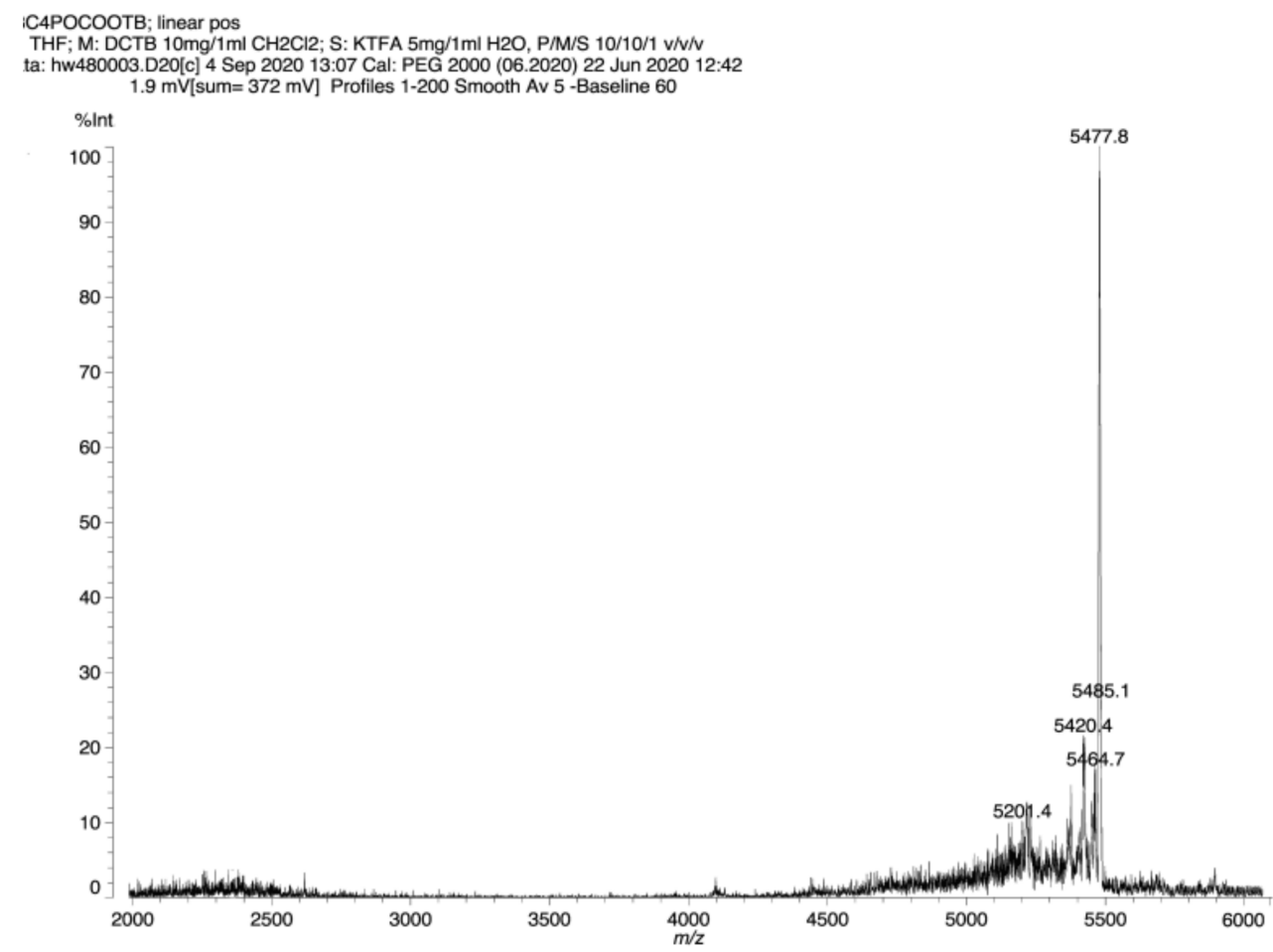

Figure 1. The MALDI TOF MS spectrum of dendrimer 10.

The minor peak (5420.4), which is 57 atom mass units in mass lower than the major one $(\mathrm{M}+\mathrm{K})$, presumably arose due to the insignificant fragmentation of one (out of 24) tert-butyl cation. The subsequent cleavage (TFA-DCM) of all tert-butyl esters (91\%) led to the formation of third generation dendrimer $\mathbf{1 1}$ with 24 free carboxyl groups, which was further converted into the corresponding polyanion 12 by the reaction with aqueous sodium bicarbonate. It is worth noting that the terminal bulky tert-butyl groups do not cause any adverse congestion effects at the surface, and the third generation dendrimer was formed smoothly.

Secondly, when catalytic hydrogenolysis could not be used during the synthesis (such as the presence of nucleophilic sulfur in the substrate), an obvious solution was the replacement of dibenzyl ester 1a with di-tert-butyl ester $\mathbf{1}$ (Scheme 2). Therefore, the reaction of another core triol, tris(4-hydroxybutyl) thiophosphate $\mathbf{1 3}[21,28]$ with an excess of di-tert-butyl trimesoate (1), again in the presence of the EDC and DMAP provided the first generation dendrimer $\mathbf{1 4}$ in $90 \%$ isolated yield. Similarly, the removal of the terminal tert-butyl ester groups from 14 occurred clearly in the TFA-DCM 3:2 mixture at room temperature. No trace of any desulfuration product was detected by means of the ${ }^{31} \mathrm{P}$ 
NMR. The hexacarboxylic acid 15 as well as its sodium salt $\mathbf{1 6}$ were obtained in high yields. The reaction of the acid 15 with an excess of the benzyl alcohol 2 (EDC, DMAP, DCM-THF 2:1) furnished another second generation polyester 17 in $84 \%$ isolated yield. Dendrimer 17 was an evident precursor of the polyanionic dendrimer 19, which was obtained by the use of tert-butyl ester cleavage in $\mathbf{1 7}$ (via polyacid 18) using the reagents and conditions mentioned above.

At last, di-tert-butyl trimesoate (1) can also be applied as a monomer for the modification of the surface of practically any small and medium-size dendrimer, terminated with nucleophilic functions such as: Hydroxyl, amino, thio, etc. Obviously, this conversion will eventually lead also to the polyanionic compound. A first generation hydroxyl-terminated, thiophosphate dendrimer 20 was synthesized using the amidophosphite approach [31-33], as described previously [21,22,28]. Therefore, the hexa-hydroxyl compound 20 possessing both four phosphorus and sulfur atoms was swiftly converted (via fully protected polyester 21) into a second generation polyanionic dendrimer precursor-dodecaacid 22 using the two-step condensation and deprotection procedure, as described above. In the end, polyacid 22 was naturally transformed into the corresponding dodecaanion $\mathbf{2 3}$ using diluted aqueous sodium bicarbonate (Scheme 3).

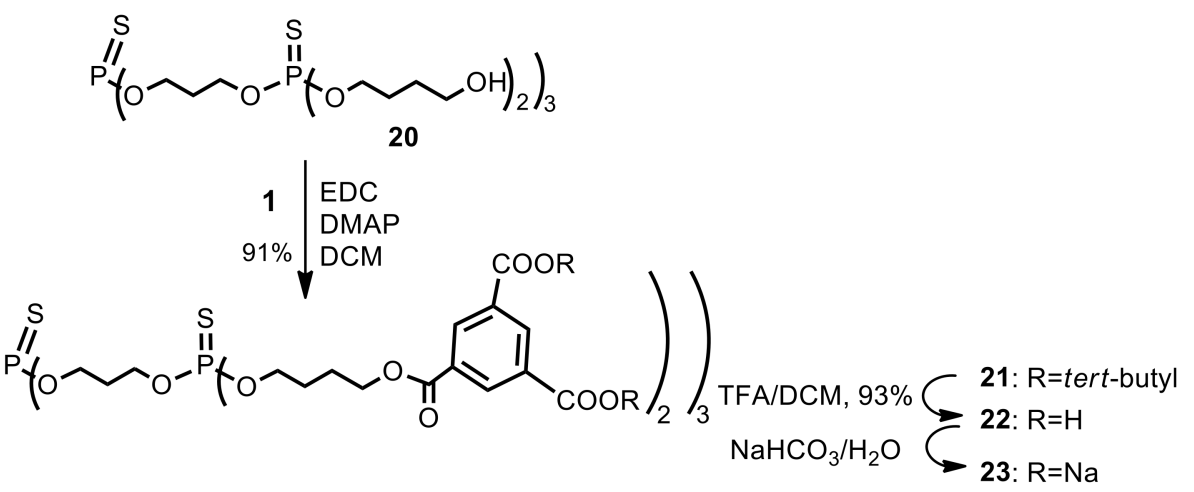

Scheme 3. Synthesis of the second generation polyanionic dendrimer 23 and its precursorsdodecacarboxylic acid 22 and polyester 21.

Dendrimers 21-23 are the examples of "mixed" or layered dendrimers, which have branching points both at phosphorus and at the carbon. In consequence, their ${ }^{1} \mathrm{H}$ and ${ }^{13} \mathrm{C}$ NMR spectra are remarkably conclusive, when compared with spectra of typical dendrimers. This is due to the diversity of the signals corresponding to nuclei absorbing in distinct areas. For instance, Figure 2 shows the ${ }^{13} \mathrm{C} N M R\left(\mathrm{CD}_{3} \mathrm{OD}-\mathrm{CDCl}_{3} 3: 1\right)$ spectrum of the polyacid 22. Signals at 26.4 and 28.1 ppm are attributed to 12 "inner" carbon atoms from four-carbon chains, whereas the signal (multiplet, coupling with two phosphorus atoms) at 30.6-30.7 (very minor) is attributed to the three "middle" carbons of the three-carbon chains. Carbon atoms, which are in alpha position to the ester moieties, resonate at $63.4 \mathrm{(m}, 6 \mathrm{C}$, three-carbon chain), 66.5 (6C, four-carbon chain), and 69.1 (doublet, 6C four-carbon chain) ppm, respectively. The aromatic range of signals looks quite clear. There are 36 carbons distributed as follows: $6 \mathrm{CH}, 12 \mathrm{CH}, 12 \mathrm{C}$ ipso, $6 \mathrm{C}$ ipso. Finally, there are six ester carbonyls (166.6 ppm) and twelve carboxylic acid carbonyls (167.9 ppm). 


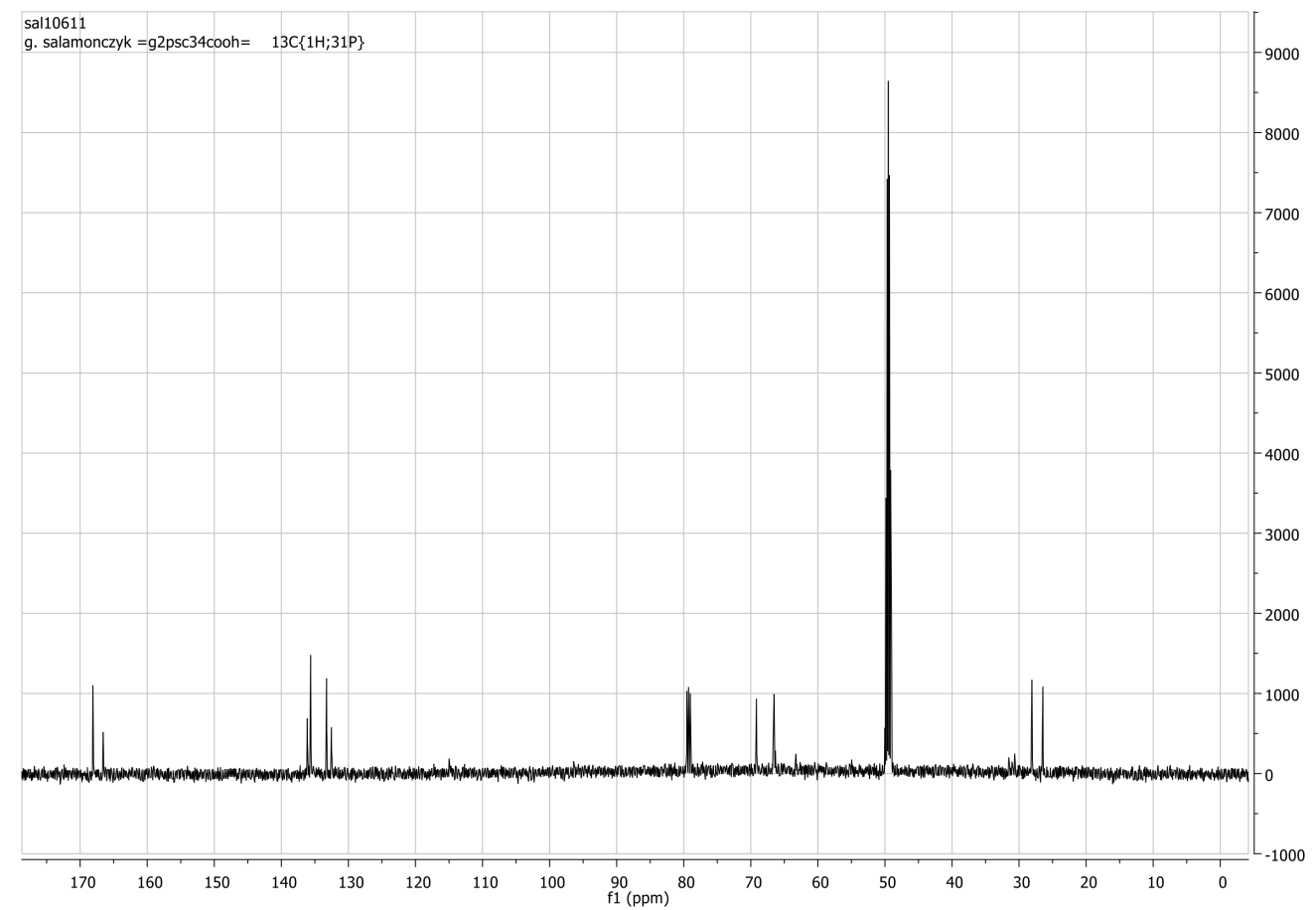

Figure 2. ${ }^{13} \mathrm{C}$ NMR spectrum of polyanionic dendrimer 22.

The synthesized polyanionic dendrimers (6-G1, 9-G2, 12-G3, 16-G1, 19-G2, 23-G2) were water-soluble non-hygroscopic white solids (powders). All of the synthesized polyanionic dendrimers possessed a decent water-solubility (at least $50 \mathrm{mg} / 1 \mathrm{~mL}$ ). They are chemically stable in the aqueous solutions, at least, at the $\mathrm{pH}$ range from 5 to 9 . However, as expected, for the macromolecular compounds, they were not stable enough for the melting point measurements. The synthesized dendrimers, as polyesters, have the biodegradability potential, which is critical for biomedical applications. These compounds will be tested against commonly known pathogenic viruses. The author also hopes that they will be tested against the SARS-2 coronavirus.

\section{Materials and Methods}

\subsection{General Procedures}

The melting points reported here are uncorrected and were determined using the MelTemp ${ }^{\circledR}$ Digital Melting Point Apparatus. The NMR spectra $\left({ }^{1} \mathrm{H},{ }^{13} \mathrm{C},{ }^{31} \mathrm{P}\right)$ were recorded on Bruker Avance AV-200, AV-500 or AV-600 spectrometers (Bruker Corporation, Billerica, MA, USA) (200, 500 or $600 \mathrm{MHz}$, respectively). Superscripts in the NMR spectra description refer to the dendrimer generation number. ${ }^{13} \mathrm{C}$ NMR spectra were assisted with DEPT 90 and DEPT 135 experiments. High resolution mass spectra were recorded on the SYNAPT G2-Si (Waters Corporation, Milford, MA, USA) spectrometer and the matrix assisted laser desorption ionization time of flight mass spectra (MALDI TOF MS) were run on the MALDI AXIMA Performance ToF/ToF (Shimadzu Corporation, Kyoto, Kyoto, Japan) mass spectrometer using 2,5-dihydroxybenzoic acid as the matrix. FT-IR spectra were measured on the ATI (American Thermal Instruments, Dayton, OH, USA) Mattson Infinity 60 AR spectrophotometer. Microanalyses were performed on EuroVector 3018 and/or on Vario (Elementar Analysensysteme GmbH, Hanau, Germany) analyzers. Chemical reagents were purchased from Sigma-Aldrich and were used without any further purification. Thin layer chromatography was carried out on Merck silica gel $60 \mathrm{~F}_{254}$ aluminum sheets using UV light $(254 \mathrm{~nm})$ or phosphomolybdic acid ( $5 \%$ solution in ethanol) for the spots visualization. Preparative flash chromatography was performed on silica gel columns (Merck, Kieselgel 230-400 mesh). The terms: "Short column" or "short pad (plug) of silica gel" used throughout this section refer to the column of silica gel with a length of $25 \mathrm{~mm}$ 
and diameter of $30 \mathrm{~mm}$. Solvents were obtained from commercial sources (usually from POCh S.A., Poland) and distilled or dried according to the standard methods. The NMR and mass spectra for the synthesized compounds are shown in Supplementary Materials.

Please note, the NMR spectra of sodium salts: 6, 9, 12, 16, 19, 23 were virtually identical with the spectra of their corresponding precursors-acids: 5, 8, 11, 15, 18, 22. Therefore, they are not reported.

\subsection{Synthetic Procedures and Analyses for the Monomers 1 and 2}

Di-tert-butyl 1,3,5-benzenetricarboxylate (1). To a solution of 1,3,5-benzenetricarbonyl trichloride $(2.65 \mathrm{~g}, 10 \mathrm{mmol})$ in dry THF $(60 \mathrm{~mL})$ at $-20^{\circ} \mathrm{C}$, was slowly (during about $1 \mathrm{~h}$ (syringe pump useful) added a $1.0 \mathrm{M}$ solution of lithium tert-butoxide (20 mL, $20 \mathrm{mmol}$, 2 equiv.) in dry THF, and the resulting mixture was stirred for an additional $1 \mathrm{~h}$, at $-20{ }^{\circ} \mathrm{C}$, then for the next $1 \mathrm{~h}$ at room temperature. Afterwards, a $2 \mathrm{M}$ aqueous solution $(15 \mathrm{~mL})$ of sodium bicarbonate was added and the mixture was stirred for $30 \mathrm{~min}$ at room temperature. Then, the THF was removed in a vacuo and the $\mathrm{H}_{2} \mathrm{O}-\mathrm{MeOH}$ mixture $(2: 1,30 \mathrm{~mL})$ was added to the residue and its $\mathrm{pH}$ was adjusted to $\sim 10$ (with $1 \mathrm{M} \mathrm{NaOH}$ ). The resulting mixture was extracted with hexane $(2 \times 20 \mathrm{~mL})$ and the hexane solutions were concentrated and the residue (tri-tert-butyl 1,3,5-benzenetricarboxylate $415 \mathrm{mg}, 11 \%$ ) was analyzed by the ${ }^{1} \mathrm{H}$ $\operatorname{NMR}\left(200 \mathrm{MHz}, \mathrm{CDCl}_{3}\right) \delta=1.52(\mathrm{~s}, 27 \mathrm{H}), 8.54$ (s, 3H) ppm.

Next, the organic volatiles were removed in vacuo once again and water $(50 \mathrm{~mL})$ was added to the residue and its $\mathrm{pH}$ was set to $\sim 8.5$ (with $0.1 \mathrm{M} \mathrm{HCl}$ ). The mixture was extracted with EtOAc $(4 \times 30 \mathrm{~mL})$. The combined EtOAc solution was concentrated. The residual material was then purified by flash chromatography using $\mathrm{CH}_{2} \mathrm{Cl}_{2}$-acetone (gradually increasing polarity from 30:1 to 20:1) as an eluent. Yield of $\mathbf{1}$ (white solid) $1.55 \mathrm{~g} \mathrm{(48 \% ).} R_{f}$ $0.15\left(\mathrm{CH}_{2} \mathrm{Cl}_{2}\right.$-acetone 20:1). Crystallization from the $\mathrm{CH}_{2} \mathrm{Cl}_{2}$-cyclohexane mixture afforded the crystalline title compound 1; mp 202-204 ${ }^{\circ} \mathrm{C}$ (decomposed); IR v max 2966, 1737, 1482, 1273, 1112, $761 \mathrm{~cm}^{-1}$; NMR: $\delta_{\mathrm{H}}(600 \mathrm{MHz}, \mathrm{CDCl} 3) 1.66(\mathrm{~s}, 18 \mathrm{H}), 8.83\left[\mathrm{t},{ }^{3} \mathrm{~J}(\mathrm{H}, \mathrm{H})=1.7 \mathrm{~Hz}\right.$, $\left.1 \mathrm{H}], 8.86\left[\mathrm{~d},{ }^{3} J(\mathrm{H}, \mathrm{H})=1.7 \mathrm{~Hz}, 2 \mathrm{H}\right] \mathrm{ppm} ; \delta_{\mathrm{C}}\left(50 \mathrm{MHz}, \mathrm{CDCl}_{3}\right) 28.11\left\{6 \mathrm{C},\left[\left(\mathrm{CH}_{3}\right)_{3} \mathrm{C}\right)\right]\right\}$, $\left.82.32\left\{2 \mathrm{C},\left[\left(\mathrm{CH}_{3}\right)_{3} \mathrm{C}\right)\right]\right\}, 129.9$ (Ar), 132.7 [2C, (Ar)], 134.6 [2C (ipso Ar)], 135.2 (ipso Ar), 164.1 [2C, $(\mathrm{C}=\mathrm{O})$ ], $170.7(\mathrm{C}=\mathrm{O})$ ppm; HRMS (APCI): $\mathrm{M}-\mathrm{H}^{+}$, found 321.1338. $\mathrm{C}_{17} \mathrm{H}_{28} \mathrm{O}_{6}$ requires 321.1338 .

The remaining aqueous solution was acidified to the $\mathrm{pH} \sim 3.0$ with $6 \mathrm{M} \mathrm{HCl}$, yielding a white dispersion. The organic material was then extracted with EtOAc $(2 \times 15 \mathrm{~mL})$. The solvent was finally removed to give tert-butyl 1.3.5-benzenetricarboxylate as a white solid (450 mg, 17\%). ${ }^{1} \mathrm{H}$ NMR (500 MHz, CD $\left.\mathrm{CD}_{3} \mathrm{CDCl}_{3} 1: 1\right) \delta=1.56(\mathrm{~s}, 9 \mathrm{H}), 8.80\left[\mathrm{~d},{ }^{4} J(\mathrm{H}, \mathrm{H})=\right.$ $1.6 \mathrm{~Hz}, 2 \mathrm{H}], 8.82\left[\mathrm{t},{ }^{4} J(\mathrm{H}, \mathrm{H})=1.6 \mathrm{~Hz}, 1 \mathrm{H}\right] \mathrm{ppm}$.

Di-tert-butyl 5-hydroxymethylbenzene-1,3-dicarboxylate (2). Di-tert-butyl 1,3,5benzenetricarboxylate (1) $(1.29 \mathrm{~g}, 4.0 \mathrm{mmol})$ was dissolved in dry THF (8 mL). The boranemethyl sulfide complex ( $4.0 \mathrm{~mL}$ of $2 \mathrm{M}$ solution in toluene, $8.0 \mathrm{mmol}$ ) was added slowly, accompanied by effervescence. After $20 \mathrm{~h}$ of stirring at room temperature, methanol $(15 \mathrm{~mL})$ was added. The solution was stirred at room temperature for $30 \mathrm{~min}$ and concentrated under reduced pressure. The resulting white solid was dissolved in ethyl acetate $(30 \mathrm{~mL})$ and washed with water $(25 \mathrm{~mL})$ and saturated $\mathrm{NaHCO}_{3}(20 \mathrm{~mL})$. The solvent was removed under reduced pressure, and the crude product was purified by a short column silica chromatography $\left(\mathrm{CH}_{2} \mathrm{Cl}_{2}\right.$-acetone $\left.50: 1\right)$ to give 2 as a white solid $(1.09 \mathrm{~g}, 88 \%) . R_{f} 0.29$ $\left(\mathrm{CH}_{2} \mathrm{Cl}_{2}\right.$-acetone 35:1). Crystallization from the $\mathrm{CH}_{2} \mathrm{Cl}_{2}$-cyclohexane mixture afforded the crystalline compound: $\mathrm{Mp} 144-145^{\circ} \mathrm{C} ;{ }^{1} \mathrm{H}$ NMR $\left(200 \mathrm{MHz}, \mathrm{CDCl}_{3}\right) \delta=1.56(\mathrm{~s}, 9 \mathrm{H}), 4.74$ $(\mathrm{s}, 2 \mathrm{H}), 8.08\left[\mathrm{~d},{ }^{4} J(\mathrm{H}, \mathrm{H})=1.6 \mathrm{~Hz}, 2 \mathrm{H}\right], 8.42\left[\mathrm{t},{ }^{4} J(\mathrm{H}, \mathrm{H})=1.6 \mathrm{~Hz}, 1 \mathrm{H}\right] \mathrm{ppm} ; \delta_{\mathrm{C}}(50 \mathrm{MHz}$, $\left.\left.\left.\mathrm{CDCl}_{3}\right) 28.63\left\{6 \mathrm{C},\left[\left(\mathrm{CH}_{3}\right)_{3} \mathrm{C}\right)\right]\right\}, 64.59\left(\underline{\mathrm{CH}}_{2} \mathrm{OH}\right), 82.20\left\{2 \mathrm{C},\left[\left(\mathrm{CH}_{3}\right)_{3} \mathrm{C}\right)\right]\right\}, 129.9(\mathrm{Ar}), 132.0[2 \mathrm{C}$, (Ar)], 132.9 [2C (ipso Ar)], 142.3 (ipso Ar), 165.7 [2C, ( $\underline{C}=\mathrm{O})$ ]; HRMS (APCI): $\mathrm{M}-\mathrm{H}^{+}$, found 307.1541. $\mathrm{C}_{17} \mathrm{H}_{28} \mathrm{O}_{6}$ requires 307.1545. 
3.3. Synthetic Procedures and Analyses for Dendrimers: 4, 5, 6, 7, 8, 9, 10, 11, 12, 13, 14, 15, 16, $17,18,19$

First generation polyester dendrimer 4. Tris(4-hydroxybutyl) phosphate 3 (FW 314.3, $190 \mathrm{mg}$, $0.6 \mathrm{mmol})$, was dispersed in dry dichloromethane $(6 \mathrm{~mL})$. Dibenzyl trimesoate $(\mathbf{1 a})(800 \mathrm{mg}$, $2.05 \mathrm{mmol}, 3.4$ equiv.) and 1-ethyl-3-(3-dimethylaminopropyl)carbodiimide (EDC) (FW 191.7, 403 mg, 2.1 mmol, 3.5 equiv.) were added under argon atmosphere. The suspension was stirred vigorously and quickly dissolved. After $5 \mathrm{~min}, 4$-dimethylaminopyridine (DMAP) (FW 122.2, $36 \mathrm{mg}, 0.3 \mathrm{mmol}, 0.4$ equiv.) was added and the mixture was stirred at room temperature overnight. The reaction mixture was then diluted with ethyl acetate $(60 \mathrm{~mL})$, washed with $0.1 \mathrm{M}$ citric acid $(15 \mathrm{~mL})$, dried $\left(\mathrm{MgSO}_{4}\right)$, and evaporated under reduced pressure. The residue was placed on a short plug of silica gel. Elution with the $\mathrm{CH}_{2} \mathrm{Cl}_{2}-\mathrm{MeOH}$ 100:1 mixture and gradually increasing the polarity to $\mathrm{CH}_{2} \mathrm{Cl}_{2}-\mathrm{MeOH} 20: 1$, gave the title ester $4(790 \mathrm{mg})$. Yield 92\%. $R_{f} 0.3\left(\mathrm{CH}_{2} \mathrm{Cl}_{2}-\mathrm{MeOH} 20: 1\right) ; \mathrm{NMR}: \delta_{\mathrm{H}}(500 \mathrm{MHz}$, $\left.\mathrm{CDCl}_{3}\right)$ 1.86-1.87 [m, $\left.{ }^{3} \mathrm{~J}(\mathrm{H}, \mathrm{H})=6.4 \mathrm{~Hz}, 6 \mathrm{H},\left(\mathrm{POCH}_{2} \mathrm{CH}_{2} \mathrm{CH}_{2} \mathrm{CH}_{2} \mathrm{OC}\right)\right], 1.91-1.92\left[\mathrm{~m},{ }^{3} J(\mathrm{H}, \mathrm{H})\right.$ $\left.=6.4 \mathrm{~Hz}, 6 \mathrm{H},\left(\mathrm{POCH}_{2} \mathrm{CH}_{2} \mathrm{CH}_{2} \mathrm{CH}_{2} \mathrm{OC}\right)\right], 4.14\left[\mathrm{dt},{ }^{3} \mathrm{~J}(\mathrm{H}, \mathrm{H})=5.8 \mathrm{~Hz},{ }^{3} J(\mathrm{P}, \mathrm{H})=11.2 \mathrm{~Hz}, 6 \mathrm{H}\right.$, $\left.\left(\mathrm{POCH}_{2} \mathrm{CH}_{2} \mathrm{CH}_{2} \mathrm{CH}_{2} \mathrm{OC}\right)\right], 4.42\left[\mathrm{t},{ }^{3} \mathrm{~J}(\mathrm{H}, \mathrm{H})=5.8 \mathrm{~Hz}, 6 \mathrm{H},\left(\mathrm{POCH}_{2} \mathrm{CH}_{2} \mathrm{CH}_{2} \mathrm{CH}_{2} \mathrm{OC}\right)\right], 5.39$ [s, $\left.12 \mathrm{H},\left(\mathrm{PhCH}_{2}\right)\right], 7.32-7.47(\mathrm{~m}, 30 \mathrm{H}, \mathrm{Ph}), 8.85\left[\mathrm{~d},{ }^{4} J(\mathrm{H}, \mathrm{H})=1.5 \mathrm{~Hz}, 6 \mathrm{H}, \mathrm{Ar}\right], 8.88\left[\mathrm{t},{ }^{4} J(\mathrm{H}, \mathrm{H})=\right.$ $1.5 \mathrm{~Hz}, 3 \mathrm{H}, \mathrm{Ar}] \mathrm{ppm} ; \delta_{\mathrm{C}}\left(125 \mathrm{MHz}_{\mathrm{CDCl}}\right) 25.05$ [3C, O=COCH $\left.\mathrm{CH}_{2} \mathrm{CH}_{2} \mathrm{CH}_{2} \mathrm{O}\right)$ ], 26.94 [d, $\left.{ }^{3} \mathrm{~J}(\mathrm{C}, \mathrm{P})=6.3 \mathrm{~Hz}, 3 \mathrm{C},\left(\mathrm{OCH}_{2} \mathrm{CH}_{2} \mathrm{CH}_{2} \mathrm{CH}_{2} \mathrm{OP}\right)\right], 65.05\left[3 \mathrm{C},\left(\mathrm{OCH}_{2} \mathrm{CH}_{2} \mathrm{CH}_{2} \mathrm{CH}_{2} \mathrm{OP}\right)\right], 67.20$ $\left[\mathrm{d},{ }^{2} \mathrm{~J}(\mathrm{C}, \mathrm{P})=5.0 \mathrm{~Hz}, 3 \mathrm{C},\left(\mathrm{OCH}_{2} \mathrm{CH}_{2} \mathrm{CH}_{2} \underline{\left.\mathrm{CH}_{2} \mathrm{OP}\right)}\right), 67.43\right.$ [6C, $\left.\left(\mathrm{PhCH}_{2}\right)\right], 128.4$ [12C, $(\mathrm{PhC} \alpha)$, 128.5 [6C, $(\mathrm{PhC} \gamma)$ ], 128.7 [12C, (PhC $\beta)], 131.3$ [6C, (ipso Ph)], 134.4 [3C, (Ar)], 134.7 [6C, (Ar)], 135.5 [9C, (ipso Ar)], 164.8 [6C, $(\underline{C}=\mathrm{O})], 164.9$ [3C, $(\underline{C}=\mathrm{O})] \mathrm{ppm} ; \delta_{\mathrm{P}}\left\{{ }^{1} \mathrm{H}\right\}(202 \mathrm{MHz}$, $\left.\mathrm{CDCl}_{3}\right)-0.51$ ppm; MALDI TOF MS calcd. for $\mathrm{C}_{81} \mathrm{H}_{75} \mathrm{O}_{22} \mathrm{P}, \mathrm{M}=1430.4$. Found $\mathrm{m} / \mathrm{z}=$ $1453.9 \mathrm{M}+\mathrm{Na}, 1469.9 \mathrm{M}+\mathrm{K}$.

Polyanionic dendrimer 6. Dendrimer 4 (FW 1431.4, $300 \mathrm{mg}, 0.21 \mathrm{mmol}$ ) was dissolved in methanol $(4 \mathrm{~mL})$ and palladium on activated charcoal $(10 \%, 100 \mathrm{mg})$ was then added. The reaction mixture was stirred under a hydrogen atmosphere for $10 \mathrm{~h}$. The catalyst was filtered off, the filtrate concentrated to dryness in vacuo to furnish analytically pure acid $5(181 \mathrm{mg}, 97 \%)$ as a white solid. NMR: $\delta_{\mathrm{H}}\left(500 \mathrm{MHz}, \mathrm{CDCl}_{3}: \mathrm{CD}_{3} \mathrm{OD} 1: 1\right)$ 1.73-1.74 [m, $\left.{ }^{3} J(\mathrm{H}, \mathrm{H})=6.4 \mathrm{~Hz}, 6 \mathrm{H},\left(\mathrm{POCH}_{2} \mathrm{CH}_{2} \mathrm{CH}_{2} \mathrm{CH}_{2} \mathrm{OC}\right)\right], 1.83-1.84\left[\mathrm{~m},{ }^{3} J(\mathrm{H}, \mathrm{H})\right.$ $\left.=6.4 \mathrm{~Hz}, 6 \mathrm{H},\left(\mathrm{POCH}_{2} \mathrm{CH}_{2} \mathrm{CH}_{2} \mathrm{CH}_{2} \mathrm{OC}\right)\right], 4.09\left[\mathrm{dt},{ }^{3} \mathrm{~J}(\mathrm{H}, \mathrm{H})=5.8 \mathrm{~Hz},{ }^{3} J(\mathrm{P}, \mathrm{H})=11.2 \mathrm{~Hz}\right.$, $\left.6 \mathrm{H},\left(\mathrm{POCH}_{2} \mathrm{CH}_{2} \mathrm{CH}_{2} \mathrm{CH}_{2} \mathrm{OC}\right)\right], 4.31\left[\mathrm{t},{ }^{3} \mathrm{~J}(\mathrm{H}, \mathrm{H})=5.8 \mathrm{~Hz}, 6 \mathrm{H},\left(\mathrm{POCH}_{2} \mathrm{CH}_{2} \mathrm{CH}_{2} \mathrm{CH}_{2} \mathrm{OC}\right)\right]$, $8.71\left[\mathrm{~d},{ }^{4} J(\mathrm{H}, \mathrm{H})=1.6 \mathrm{~Hz}, 6 \mathrm{H}, \mathrm{Ar}\right], 8.74\left[\mathrm{t},{ }^{4} J(\mathrm{H}, \mathrm{H})=1.6 \mathrm{~Hz}, 3 \mathrm{H}, \mathrm{Ar}\right] \mathrm{ppm} ; \delta_{\mathrm{C}}\left\{{ }^{1} \mathrm{H}\right.$, $\left.{ }^{31} \mathrm{P}\right\}\left(125 \mathrm{MHz}, \mathrm{CD}_{3} \mathrm{OD}-\mathrm{CDCl}_{3}\right.$ 2:1) 24.35 [3C, O=COCH$\left.{ }_{2} \mathrm{CH}_{2} \mathrm{CH}_{2} \mathrm{CH}_{2} \mathrm{O}\right)$ ], 26.00 [3C, $\left.\left(\mathrm{OCH}_{2} \mathrm{CH}_{2} \mathrm{CH}_{2} \mathrm{CH}_{2} \mathrm{OP}\right)\right], 64.45$ [3C, $\left.\left(\mathrm{OCH}_{2} \mathrm{CH}_{2} \mathrm{CH}_{2} \mathrm{CH}_{2} \mathrm{OP}\right)\right], 67.07,130.4$ [3C, (ipso Ar)], 131.2 [6C, (ipso Ar)], 133.5 [6C, (Ar)], 134.0 [3C, (Ar)], 164.5 [3C, $(\underline{C}=\mathrm{O})], 166.0$ [6C, $(\underline{\mathrm{C}}=\mathrm{O})]$ ppm; $\delta_{\mathrm{P}}\left\{{ }^{1} \mathrm{H}\right\}\left(202 \mathrm{MHz}, \mathrm{CD}_{3} \mathrm{OD}-\mathrm{CDCl}_{3}\right.$ 2:1) -0.42 ppm; MALDI TOF MS calcd. for $\mathrm{C}_{39} \mathrm{H}_{39} \mathrm{O}_{22} \mathrm{P}, \mathrm{M}=890.2$. Found $\mathrm{m} / \mathrm{z}=890.2$. Next, acid $5(60 \mathrm{mg}, 0.066 \mathrm{mmol})$ was dispersed in water $(2 \mathrm{~mL})$ and solid sodium bicarbonate $(34 \mathrm{mg}, 0.4 \mathrm{mmol}, 6$ equiv.) was added. After $20 \mathrm{~min}$, the resulting solution was frozen and lyophilized under high vacuum $(0.1 \mathrm{mmHg})$ to give 6 as a hexasodium salt of $5(68 \mathrm{mg})$, as a nonhygroscopic white powder.

Second generation polyester dendrimer 7. Carboxy-terminated, first generation phosphate dendrimer 5 (FW 890.7, $100 \mathrm{mg}, 0.112 \mathrm{mmol})$, was dissolved in dry THF (2 mL). Then, the reaction mixture was diluted with dry dichloromethane (2 mL). Di-tert-butyl 5hydroxymethylbenzene-1,3-dicarboxylate (2) (FW 308.4, $230 \mathrm{mg}, 0.74 \mathrm{mmol}, 6.6$ equiv.) and 1-ethyl-3-(3-dimethylaminopropyl)carbodiimide (EDC) (172 mg, $0.896 \mathrm{mmol}, 8.0$ equiv.) were added under argon atmosphere. The suspension was stirred vigorously and quickly dissolved. After $5 \mathrm{~min}$, 4-dimethylaminopyridine (DMAP) (15 mg, $0.12 \mathrm{mmol}, 1.0$ equiv.) was added and the mixture was stirred at room temperature overnight. The reaction mixture was then diluted with ethyl acetate $(50 \mathrm{~mL})$, washed with $0.1 \mathrm{M}$ citric acid $(10 \mathrm{~mL})$, dried $\left(\mathrm{MgSO}_{4}\right)$, and evaporated under reduced pressure. The residue was placed on a short plug of silica gel. Elution with the cyclohexane-acetone 20:1 mixture and gradually increasing the polarity to cyclohexane-acetone 8:1, gave the title ester 5 (260 $\mathrm{mg})$. Yield 88\%. NMR: $\delta_{\mathrm{H}}\left(500 \mathrm{MHz}, \mathrm{CDCl}_{3}-\mathrm{C}_{6} \mathrm{D}_{6} 2: 1\right) 1.58\left[\mathrm{~s}, 108 \mathrm{H}, \mathrm{C}\left(\mathrm{CH}_{3}\right)_{3}\right], 1.81-1.86[\mathrm{~m}, 6 \mathrm{H}$, 
$\left.\left(\mathrm{POCH}_{2} \mathrm{CH}_{2} \mathrm{CH}_{2} \mathrm{CH}_{2} \mathrm{OC}\right)\right], 1.87-1.96$ [m, 6H, $\left.\left(\mathrm{POCH}_{2} \mathrm{CH}_{2} \mathrm{CH}_{2} \mathrm{CH}_{2} \mathrm{OC}\right)\right], 4.13$ [dt, ${ }^{3} J(\mathrm{H}, \mathrm{H})$ $\left.=6.3 \mathrm{~Hz},{ }^{3} \mathrm{~J}(\mathrm{P}, \mathrm{H})=12.3 \mathrm{~Hz}, 6 \mathrm{H},\left(\mathrm{POCH}_{2} \mathrm{CH}_{2} \mathrm{CH}_{2} \mathrm{CH}_{2} \mathrm{OC}\right)\right], 4.40\left[\mathrm{t},{ }^{3} \mathrm{~J}(\mathrm{H}, \mathrm{H})=6.5 \mathrm{~Hz}, 6 \mathrm{H}\right.$, $\left.\left(\mathrm{POCH}_{2} \mathrm{CH}_{2} \mathrm{CH}_{2} \mathrm{CH}_{2} \mathrm{OC}\right)\right], 5.29$ [s, 12H, $\left.\left(\mathrm{ArCH}_{2}\right)\right], 8.20\left[\mathrm{~d},{ }^{4} J(\mathrm{H}, \mathrm{H})=1.3 \mathrm{~Hz}, 12 \mathrm{H}, \mathrm{Ar}^{2}\right.$ ], $8.53\left[\mathrm{t},{ }^{4} J(\mathrm{H}, \mathrm{H})=1.3 \mathrm{~Hz}, 6 \mathrm{H}, \mathrm{Ar}^{2}\right], 8.85\left[\mathrm{~d},{ }^{3} J(\mathrm{H}, \mathrm{H})=1.5 \mathrm{~Hz}, 6 \mathrm{H}, \mathrm{Ar}^{1}\right], 8.87\left[\mathrm{t},{ }^{3} J(\mathrm{H}, \mathrm{H})\right.$ $\left.=1.5 \mathrm{~Hz}, 3 \mathrm{H}, \mathrm{Ar}^{1}\right] \mathrm{ppm} ; \delta_{\mathrm{C}}\left(125 \mathrm{MHz}, \mathrm{CDCl}_{3}\right) 25.04\left[3 \mathrm{C}, \mathrm{O}=\mathrm{COCH}_{2} \mathrm{CH}_{2} \mathrm{CH}_{2} \mathrm{CH}_{2} \mathrm{O}\right)$ ], $26.96\left[\mathrm{~d},{ }^{3} \mathrm{~J}(\mathrm{C}, \mathrm{P})=7.1 \mathrm{~Hz}, 3 \mathrm{C},\left(\mathrm{OCH}_{2} \mathrm{CH}_{2} \mathrm{CH}_{2} \mathrm{CH}_{2} \mathrm{OP}\right)\right], 28.21\left[36 \mathrm{C}, \mathrm{C}\left(\mathrm{CH}_{3}\right)_{3}\right], 65.16$ [3C, $\left.\left(\mathrm{OCH}_{2} \mathrm{CH}_{2} \mathrm{CH}_{2} \mathrm{CH}_{2} \mathrm{OP}\right)\right], 67.43\left[6 \mathrm{C},\left(\mathrm{ArCH}_{2}\right)\right], 67.20\left[\mathrm{~d},{ }^{2} J(\mathrm{C}, \mathrm{P})=5.7 \mathrm{~Hz}, 3 \mathrm{C}\right.$, $\left(\mathrm{OCH}_{2} \mathrm{CH}_{2} \mathrm{CH}_{2} \mathrm{CH}_{2} \mathrm{OP}\right)$ ], 81.92 [12C, $\mathrm{C}\left(\mathrm{CH}_{3}\right)_{3}$ ], 130.6 [6C, $\left(\mathrm{Ar}^{2}\right)$ ], 131.1 [6C, (ipso $\left.\mathrm{Ar}^{1}\right)$ ], 131.5 [3C, $\left(\right.$ ipso $\left.\mathrm{Ar}^{1}\right)$ ], 132.9 [12C, $\left(\right.$ ipso $\left.\mathrm{Ar}^{2}\right)$ ], 133.2 [12C, $\left(\mathrm{Ar}^{2}\right)$ ], 134.9 [3C, $\left(\mathrm{Ar}^{1}\right)$ ], 135.0 [6C, $\left(\mathrm{Ar}^{1}\right)$ ], 136.1 [6C, $\left(\right.$ ipso $\left.\mathrm{Ar}^{2}\right)$ ], 164.7 [18C, $\left.(\underline{\mathrm{C}}=\mathrm{O})\right], 164.8$ [3C, $\left.(\underline{\mathrm{C}}=\mathrm{O})\right]$ ppm; $\delta_{\mathrm{P}}\left\{{ }^{1} \mathrm{H}\right\}(202 \mathrm{MHz}$, $\left.\mathrm{CDCl}_{3}\right) 0.53$ ppm; MALDI TOF MS calcd. for $\mathrm{C}_{141} \mathrm{H}_{171} \mathrm{O}_{46} \mathrm{P}, \mathrm{M}=2631.1$. Found $\mathrm{m} / \mathrm{z}=$ $2670.3 \mathrm{M}+\mathrm{K}$.

Polyanionic dendrimer 9. Dendrimer 7 (FW 2632.8, $134 \mathrm{mg}, 0.05 \mathrm{mmol}$ ) was dissolved in dry dichloromethane $(2 \mathrm{~mL})$, and trifluoroacetic acid was added $(2 \mathrm{~mL})$. The reaction mixture was stirred at room temperature under argon atmosphere for $5 \mathrm{~h}$. All the volatiles were removed in vacuo, and the residue was dissolved in THF $(1 \mathrm{~mL})$. Next, acetone $(10 \mathrm{~mL})$ was added to that solution and the resulting mixture was kept in the refrigerator for about an hour. The precipitate was filtered off to provide acid $8(88 \mathrm{mg}, 90 \%)$ as a white solid. NMR: $\delta_{\mathrm{H}}\left(500 \mathrm{MHz}, \mathrm{CDCl}_{3}-\mathrm{CD}_{3} \mathrm{OD}\right.$ 2:1) 2.03-2.08 [m, 6H, $\left.\left(\mathrm{POCH}_{2} \mathrm{CH}_{2} \mathrm{CH}_{2} \mathrm{CH}_{2} \mathrm{OC}\right)\right], 2.25-2.28$ $\left[\mathrm{m}, 6 \mathrm{H},\left(\mathrm{POCH}_{2} \mathrm{CH}_{2} \mathrm{CH}_{2} \mathrm{CH}_{2} \mathrm{OC}\right)\right], 3.90-3.92\left[\mathrm{~m}, 6 \mathrm{H},\left(\mathrm{POCH}_{2} \mathrm{CH}_{2} \mathrm{CH}_{2} \mathrm{CH}_{2} \mathrm{OC}\right)\right], 4.14-4.16$ $\left[\mathrm{m}, 6 \mathrm{H},\left(\mathrm{POCH}_{2} \mathrm{CH}_{2} \mathrm{CH}_{2} \mathrm{CH}_{2} \mathrm{OC}\right)\right], 5.22\left[\mathrm{~s}, 12 \mathrm{H},\left(\mathrm{ArCH}_{2}\right)\right], 8.36\left[\mathrm{~s}, 6 \mathrm{H}, \mathrm{Ar}^{2}\right], 8.48[\mathrm{~s}, 12 \mathrm{H}$, $\mathrm{Ar}^{2}$ ], $8.56\left[\mathrm{~s}, 6 \mathrm{H}, \mathrm{Ar}^{1}\right], 8.61\left[\mathrm{~s}, 3 \mathrm{H}, \mathrm{Ar}^{1}\right] \mathrm{ppm} ; \delta_{\mathrm{P}}\left\{{ }^{1} \mathrm{H}\right\}\left(202 \mathrm{MHz}, \mathrm{CD}_{3} \mathrm{OD}-\mathrm{CDCl}_{3}\right.$ 2:1) - 2.80 ppm; MALDI TOF MS calcd. for $\mathrm{C}_{93} \mathrm{H}_{75} \mathrm{O}_{46} \mathrm{P}, \mathrm{M}=1959.3$. Found $m / z=1982.6 \mathrm{M}+\mathrm{Na}$. Later on, acid 8 (35 mg, $0.0179 \mathrm{mmol})$ was dispersed in water $(2 \mathrm{~mL})$ and solid sodium bicarbonate (18 mg, $0.2 \mathrm{mmol}, 12$ equiv.) was added. After $20 \mathrm{~min}$, the resulting solution was frozen and lyophilized under high vacuum $(0.1 \mathrm{mmHg})$ to give 9 as a dodecasodium salt of 8 (40 mg), as a nonhygroscopic white powder.

Third generation polyester dendrimer 10. Carboxy-terminated, second generation phosphate dendrimer 8 (FW 1959.5, $40 \mathrm{mg}, 0.02 \mathrm{mmol}$ ), was dissolved in dry DMF (1.5 mL). Then, the resulting solution was diluted with dry dichloromethane $(2 \mathrm{~mL})$. Di-tert-butyl 5hydroxymethylbenzene-1,3-dicarboxylate (2) (FW 308.4, 82 mg, 0.264 mmol, 13.2 equiv.) and 1-ethyl-3-(3-dimethylaminopropyl)carbodiimide (EDC) (172 mg, $0.3 \mathrm{mmol}, 15$ equiv.) were added under argon atmosphere. The suspension was stirred vigorously and quickly dissolved. After $5 \mathrm{~min}$, 4-dimethylaminopyridine (DMAP) (5 mg, $0.04 \mathrm{mmol}, 1.8$ equiv.) was added and the mixture was stirred at room temperature overnight. The reaction mixture was then diluted with ethyl acetate $(50 \mathrm{~mL})$, washed with $0.1 \mathrm{M}$ citric acid $(10 \mathrm{~mL})$, dried $\left(\mathrm{MgSO}_{4}\right)$, and evaporated under reduced pressure. The residue was placed on a short plug of silica gel. Elution with the cyclohexane-acetone 20:1 mixture and gradually increasing the polarity to cyclohexane-acetone $6: 1$, provided the title ester 10 (83 mg). Yield 76\%. NMR: $\delta_{\mathrm{H}}\left(500 \mathrm{MHz}, \mathrm{CDCl}_{3}\right) 1.56\left[\mathrm{~s}, 216 \mathrm{H}, \mathrm{C}\left(\mathrm{CH}_{3}\right)_{3}\right] 1.82-1.87$ [m, 6H, $\left.\left(\mathrm{POCH}_{2} \mathrm{CH}_{2} \mathrm{CH}_{2} \mathrm{CH}_{2} \mathrm{OC}\right)\right], 1.88-1.95\left[\mathrm{~m}, 6 \mathrm{H},\left(\mathrm{POCH}_{2} \mathrm{CH}_{2} \mathrm{CH}_{2} \mathrm{CH}_{2} \mathrm{OC}\right)\right], 4.13$ [dt, $\left.{ }^{3} J(\mathrm{H}, \mathrm{H})=5.9 \mathrm{~Hz},{ }^{3} \mathrm{~J}(\mathrm{P}, \mathrm{H})=12.2 \mathrm{~Hz}, 6 \mathrm{H},\left(\mathrm{POCH}_{2} \mathrm{CH}_{2} \mathrm{CH}_{2} \mathrm{CH}_{2} \mathrm{OC}\right)\right], 4.39\left[\mathrm{t},{ }^{3} J(\mathrm{H}, \mathrm{H})=\right.$ $\left.6.4 \mathrm{~Hz}, 6 \mathrm{H},\left(\mathrm{POCH}_{2} \mathrm{CH}_{2} \mathrm{CH}_{2} \mathrm{CH}_{2} \mathrm{OC}\right)\right], 5.44$ [s, $\left.24 \mathrm{H},\left(\mathrm{Ar}^{3} \mathrm{CH}_{2}\right)\right], 5.46\left[\mathrm{~s}, 12 \mathrm{H},\left(\mathrm{Ar}^{2} \mathrm{CH}_{2}\right)\right]$, $8.19\left[\mathrm{~s}, 24 \mathrm{H}, \mathrm{Ar}^{3}\right], 8.35\left[\mathrm{~s}, 12 \mathrm{H}, \mathrm{Ar}^{3}\right], 8.51\left[\mathrm{~s}, 12 \mathrm{H}, \mathrm{Ar}^{2}\right], 8.69\left[\mathrm{~s}, 6 \mathrm{H}^{2} \mathrm{Ar}^{2}\right], 8.83[\mathrm{~s}, 6 \mathrm{H}$, $\mathrm{Ar}^{1}$ ], $8.85\left[\mathrm{~s}, 3 \mathrm{H}, \mathrm{Ar}^{1}\right.$ ] ppm; $\delta_{\mathrm{C}}\left(125 \mathrm{MHz}^{\mathrm{C}} \mathrm{CDCl}_{3}\right) 24.94$ [3C, $\left(\mathrm{O}=\mathrm{COCH}_{2} \mathrm{CH}_{2} \mathrm{CH}_{2} \mathrm{CH}_{2} \mathrm{O}\right)$ ], $26.91\left[\mathrm{~d},{ }^{3} \mathrm{~J}(\mathrm{C}, \mathrm{P})=7.4 \mathrm{~Hz}, 3 \mathrm{C},\left(\mathrm{OCH}_{2} \mathrm{CH}_{2} \underline{\mathrm{CH}}_{2} \mathrm{CH}_{2} \mathrm{OP}\right)\right], 28.14\left[72 \mathrm{C}, \mathrm{C}\left(\underline{\mathrm{CH}}_{3}\right)_{3}\right], 65.12[3 \mathrm{C}$, $\left(\mathrm{OCH}_{2} \mathrm{CH}_{2} \mathrm{CH}_{2} \mathrm{CH}_{2} \mathrm{OP}\right)$ ], 66.27 [6C, $\left(\mathrm{Ar}^{2} \underline{\mathrm{C}} \mathrm{H}_{2}\right)$ ], 66.33 [12C, $\left.\left(\mathrm{Ar}^{3} \mathrm{CH}_{2}\right)\right], 67.09$ [d, ${ }^{2} \mathrm{~J}(\mathrm{C}, \mathrm{P})$ $=6.7 \mathrm{~Hz}, 3 \mathrm{C},\left(\mathrm{OCH}_{2} \mathrm{CH}_{2} \mathrm{CH}_{2} \mathrm{CH}_{2} \mathrm{OP}\right)$ ], 81.81 [24C, $\left.\mathrm{C}\left(\mathrm{CH}_{3}\right)_{3}\right], 130.5$ [12C, $\left.\left(\mathrm{Ar}^{3}\right)\right], 131.0$ [6C, $\left(\mathrm{Ar}^{2}\right)$ ], 133.0 [24C, $\left(\mathrm{Ar}^{3}\right)$ ] 130.5 [12C, $\left(\mathrm{Ar}^{3}\right)$ ], 130.8 [6C, (ipso $\left.\mathrm{Ar}^{1}\right)$ ], 131.0 [12C, (ipso $\left.\mathrm{Ar}^{2}\right)$ ], 131.5 [3C, (ipso $\left.\mathrm{Ar}^{1}\right)$ ], 132.8 [24C, (ipso $\left.\mathrm{Ar}^{3}\right)$ ], 134.2 [12C, $\left(\mathrm{Ar}^{2}\right)$ ], 135.0 [9C, $\left(\mathrm{Ar}^{1}\right)$ ], 136.2 [12C, (ipso $\left.\mathrm{Ar}^{3}\right)$ ], 136.7 [6C, $\left(\right.$ ipso $\left.\left.\mathrm{Ar}^{2}\right)\right], 164.5$ [9C, $\left.\left(\mathrm{C}^{1}=\mathrm{O}\right)\right], 164.6\left[24 \mathrm{C},\left(\mathrm{C}^{3}=\mathrm{O}\right)\right], 165.0$ [12C, $\left.\left(\mathrm{C}^{2}=\mathrm{O}\right)\right] \mathrm{ppm} ; \delta_{\mathrm{P}}\left\{{ }^{1} \mathrm{H}\right\}\left(202 \mathrm{MHz}, \mathrm{CDCl}_{3}\right)-0.46 \mathrm{ppm}$. MALDI TOF MS calcd. for $\mathrm{C}_{297} \mathrm{H}_{339} \mathrm{O}_{94} \mathrm{P}, \mathrm{M}=5440.1$. Found $m / z=5477.8 \mathrm{M}+\mathrm{K}$. 
Polyanionic dendrimer 12. Dendrimer $10(80 \mathrm{mg}, 0.015 \mathrm{mmol})$ was dissolved in dry dichloromethane $(1.5 \mathrm{~mL})$, and trifluoroacetic acid was added $(2 \mathrm{~mL})$. The reaction mixture was stirred at room temperature under argon atmosphere for $5 \mathrm{~h}$. All the volatiles were removed in vacuo, and the residue was dissolved in THF $(1 \mathrm{~mL})$. Next, acetone $(10 \mathrm{~mL})$ was added to that solution and the resulting mixture was kept in the refrigerator for about an hour. The precipitate was filtered off to provide acid $11(54 \mathrm{mg}, 88 \%)$ as a white solid. NMR: $\delta_{\mathrm{H}}\left(500 \mathrm{MHz}, \mathrm{THF}-\mathrm{d}_{8}\right) 1.75-1.80\left[\mathrm{~m}, 6 \mathrm{H},\left(\mathrm{POCH}_{2} \mathrm{CH}_{2} \mathrm{CH}_{2} \mathrm{CH}_{2} \mathrm{OC}\right)\right], 1.82-1.88\left[\mathrm{~m},{ }^{3} \mathrm{~J}(\mathrm{H}, \mathrm{H})\right.$ $\left.=6.4 \mathrm{~Hz}, 6 \mathrm{H},\left(\mathrm{POCH}_{2} \mathrm{CH}_{2} \mathrm{CH}_{2} \mathrm{CH}_{2} \mathrm{OC}\right)\right], 4.07\left[\mathrm{dt}^{3}{ }^{3}(\mathrm{P}, \mathrm{H})=12.3 \mathrm{~Hz},{ }^{3} \mathrm{~J}(\mathrm{H}, \mathrm{H})=6.2 \mathrm{~Hz}, 6 \mathrm{H}\right.$, $\left.\left(\mathrm{POCH}_{2} \mathrm{CH}_{2} \mathrm{CH}_{2} \mathrm{CH}_{2} \mathrm{OC}\right)\right], 4.33\left[\mathrm{t},{ }^{3} \mathrm{~J}(\mathrm{H}, \mathrm{H})=6.2 \mathrm{~Hz}, 6 \mathrm{H},\left(\mathrm{POCH}_{2} \mathrm{CH}_{2} \mathrm{CH}_{2} \mathrm{CH}_{2} \mathrm{OC}\right)\right], 5.48[\mathrm{~s}$, $\left.24 \mathrm{H},\left(\mathrm{Ar}^{3} \mathrm{CH}_{2}\right)\right], 5.49\left[\mathrm{~s}, 12 \mathrm{H},\left(\mathrm{Ar}^{2} \mathrm{CH}_{2}\right)\right], 8.30\left[\mathrm{~d},{ }^{4} J(\mathrm{H}, \mathrm{H})=1.3 \mathrm{~Hz}, 33 \mathrm{H}, 9 \mathrm{HAr}^{1}, 24 \mathrm{HAr}^{3}\right]$, $8.58\left[\mathrm{~s}, 12 \mathrm{H}, \mathrm{Ar}^{3}\right], 8.76\left[\mathrm{~d},{ }^{4} \mathrm{~J}(\mathrm{H}, \mathrm{H})=1.6 \mathrm{~Hz}, 12 \mathrm{H}, \mathrm{Ar}^{2}\right], 8.81\left[\mathrm{t},{ }^{4} \mathrm{~J}(\mathrm{H}, \mathrm{H})=1.5 \mathrm{~Hz}, 6 \mathrm{H}, \mathrm{Ar}^{2}\right]$ ppm; $\delta_{\mathrm{C}}\left(125 \mathrm{MHz}, \mathrm{THF}-\mathrm{d}_{8}\right) 26.60\left[\mathrm{~d},{ }^{3} J(\mathrm{C}, \mathrm{P})=7.2 \mathrm{~Hz}, 3 \mathrm{C},\left(\mathrm{OCH}_{2} \mathrm{CH}_{2} \mathrm{CH}_{2} \mathrm{CH}_{2} \mathrm{OP}\right)\right], 29.24$ [3C, $\left(\mathrm{O}=\mathrm{COCH}_{2} \mathrm{CH}_{2} \mathrm{CH}_{2} \mathrm{CH}_{2} \mathrm{O}\right)$ ], 64.84 [6C, $\left.\left(\mathrm{Ar}^{2} \mathrm{CH}_{2}\right)\right], 66.08$ [12C, $\left(\mathrm{Ar}^{3} \mathrm{CH}_{2}\right)$ ], 67.09 [3C, $\left.\left(\mathrm{OCH}_{2} \mathrm{CH}_{2} \mathrm{CH}_{2} \mathrm{CH}_{2} \mathrm{OP}\right)\right], 67.34\left[\mathrm{~d},{ }^{2} \mathrm{~J}(\mathrm{C}, \mathrm{P})=6.7 \mathrm{~Hz}, 3 \mathrm{C},\left(\mathrm{OCH}_{2} \mathrm{CH}_{2} \mathrm{CH}_{2} \mathrm{CH}_{2} \mathrm{OP}\right)\right], 130.6$ [12C, $\left.\left(\mathrm{Ar}^{3}\right)\right], 131.1$ [12C, $\left.\left(\mathrm{Ar}^{2}\right)\right], 131.4$ [6C, $\left.\left(\mathrm{Ar}^{2}\right)\right], 131.7$ [24C, (ipso $\left.\left.\mathrm{Ar}^{3}\right)\right], 133.3$ [12C, (ipso $\left.\mathrm{Ar}^{2}\right)$ ], 133.3 [6C, $\left.\left(\mathrm{Ar}^{1}\right)\right], 133.4$ [24C, $\left(\mathrm{Ar}^{3}\right)$ ], 133.6 [3C, $\left(\mathrm{Ar}^{1}\right)$ ], 134.1 [6C, $\left(\right.$ ipso $\left.\left.\mathrm{Ar}^{2}\right)\right], 134.2$ [12C, (ipso $\left.\mathrm{Ar}^{3}\right)$ ], 136.8 [9C, $\left.\left(\mathrm{Ar}^{1}\right)\right], 164.0$ [12C, $\left.\left(\underline{C^{2}}=\mathrm{O}\right)\right], 164.1$ [9C, $\left.\left(\underline{\mathrm{C}^{1}}=\mathrm{O}\right)\right], 165.9$ [24C, $\left.\left(\underline{\mathrm{C}^{3}}=\mathrm{O}\right)\right]$ ppm; $\delta_{\mathrm{P}}\left\{{ }^{1} \mathrm{H}\right\}\left(202 \mathrm{MHz}, \mathrm{THF}-\mathrm{d}_{8}\right)-0.95 \mathrm{ppm}$; MALDI TOF MS calcd. for $\mathrm{C}_{201} \mathrm{H}_{147} \mathrm{O}_{94} \mathrm{P}$, $\mathrm{M}=4096.7 .3$. Found $m / z$, fragmentation: 4129.6, 3965.6, 3949.9, 3844.2, 3731.4, 3700.0, 3601.9 (major peaks). Elemental analysis: Found (acid): $\mathrm{C}, 58.87 ; \mathrm{H}, 3.66 ; \mathrm{C}_{201} \mathrm{H}_{147} \mathrm{O}_{94} \mathrm{P}$ requires C, 58.92; H, 3.62; P, 0.76\%. Afterwards, acid 11 (FW 4097.2, $40 \mathrm{mg}, 0.0098 \mathrm{mmol}$ ) was dispersed in water $(2 \mathrm{~mL})$ and solid sodium bicarbonate $(20 \mathrm{mg}, 0.235 \mathrm{mmol}, 24$ equiv.) was added. After $20 \mathrm{~min}$, the resulting solution was frozen and lyophilized under high vacuum $(0.1 \mathrm{mmHg})$ to give $\mathbf{1 2}$ as a tetracosasodium salt of $\mathbf{1 1}(45 \mathrm{mg})$, as a nonhygroscopic white powder.

First generation polyester thiophosphate dendrimer 14. Tris(4-hydroxybutyl) thiophosphate $\mathbf{1 3}$ (FW 330.4, $166 \mathrm{mg}, 0.5 \mathrm{mmol}$ ), was dissolved in dry dichloromethane (5 mL). Di-tert-butyl trimesoate (1) (322.4, $560 \mathrm{mg}, 1.7 \mathrm{mmol}, 3.4$ equiv.) and 1-ethyl-3-(3-dimethylaminopropyl) carbodiimide (EDC) (FW 191.7, $336 \mathrm{mg}, 1.75 \mathrm{mmol}$, 3.5 equiv.) were added under argon atmosphere. The suspension was stirred vigorously and quickly dissolved. After $5 \mathrm{~min}$, 4-dimethylaminopyridine (DMAP) (FW 122.2, $25 \mathrm{mg}, 0.2 \mathrm{mmol}, 0.4$ equiv.) was added and the mixture was stirred at room temperature overnight. The reaction mixture was then diluted with ethyl acetate $(60 \mathrm{~mL})$, washed with $0.1 \mathrm{M}$ citric acid $(15 \mathrm{~mL})$, dried $\left(\mathrm{MgSO}_{4}\right)$, and evaporated under reduced pressure. The residue was placed on a short plug of silica gel. Elution with the cyclohexane-acetone 100:1 mixture and gradually increasing the polarity to cyclohexane-acetone 20:1, gave the title ester $14\left(560 \mathrm{mg}\right.$ ). Yield $90 \% . R_{f} 0.25$ (cyclohexaneacetone 25:1). NMR: $\delta_{\mathrm{H}}\left(500 \mathrm{MHz}, \mathrm{CDCl}_{3}\right) 1.59\left[\mathrm{~s}, 54 \mathrm{H}, \mathrm{C}\left(\mathrm{CH}_{3}\right)_{3}\right], 1.79-1.84\left[\mathrm{~m},{ }^{3} J(\mathrm{H}, \mathrm{H})=\right.$ $6.4 \mathrm{~Hz}, 12 \mathrm{H},\left(\mathrm{POCH}_{2} \mathrm{CH}_{2} \mathrm{CH}_{2} \mathrm{CH}_{2} \mathrm{OC}\right)$ and $\left.\left(\mathrm{POCH}_{2} \mathrm{CH}_{2} \mathrm{CH}_{2} \mathrm{CH}_{2} \mathrm{OC}\right)\right], 4.12\left[\mathrm{dt},{ }^{3} J(\mathrm{H}, \mathrm{H})\right.$ $\left.=5.8 \mathrm{~Hz},{ }^{3} \mathrm{~J}(\mathrm{P}, \mathrm{H})=12.6 \mathrm{~Hz}, 6 \mathrm{H},\left(\mathrm{POCH}_{2} \mathrm{CH}_{2} \mathrm{CH}_{2} \mathrm{CH}_{2} \mathrm{OC}\right)\right], 4.42\left[\mathrm{t},{ }^{3} \mathrm{~J}(\mathrm{H}, \mathrm{H})=5.7 \mathrm{~Hz}, 6 \mathrm{H}\right.$, $\left.\left(\mathrm{POCH}_{2} \mathrm{CH}_{2} \mathrm{CH}_{2} \mathrm{CH}_{2} \mathrm{OC}\right)\right], 8.70[\mathrm{~s}, 3 \mathrm{H}, \mathrm{Ar}], 8.71[\mathrm{~s}, 6 \mathrm{H}, \mathrm{Ar}] \mathrm{ppm} ; \delta_{\mathrm{C}}\left(125 \mathrm{MHz}, \mathrm{CDCl}_{3}\right) 25.13$ [3C, O=COCH $\left.\left.\mathrm{CH}_{2} \mathrm{CH}_{2} \mathrm{CH}_{2} \mathrm{O}\right)\right], 26.77$ [d, $\left.{ }^{3} \mathrm{~J}(\mathrm{C}, \mathrm{P})=7.8 \mathrm{~Hz}, 3 \mathrm{C},\left(\mathrm{OCH}_{2} \mathrm{CH}_{2} \mathrm{CH}_{2} \mathrm{CH}_{2} \mathrm{OP}\right)\right]$, $28.19\left[18 \mathrm{C}, \mathrm{C}\left(\underline{\mathrm{CH}}_{3}\right)_{3}\right], 64.93\left[3 \mathrm{C},\left(\mathrm{OCH}_{2} \mathrm{CH}_{2} \mathrm{CH}_{2} \mathrm{CH}_{2} \mathrm{OP}\right)\right], 67.70\left[\mathrm{~d},{ }^{2} \mathrm{~J}(\mathrm{C}, \mathrm{P})=5.4 \mathrm{~Hz}, 3 \mathrm{C}\right.$, $\left(\mathrm{OCH}_{2} \mathrm{CH}_{2} \mathrm{CH}_{2} \mathrm{CH}_{2} \mathrm{OP}\right)$ ], 82.24 [6C, $\underline{\mathrm{C}}\left(\mathrm{CH}_{3}\right)_{3}$ ], 130.9 [3C, (ipso $\left.\left.\mathrm{Ar}\right)\right], 132.8$ [6C, (ipso $\left.\mathrm{Ar}\right)$ ], 134.0 [6C, $(\mathrm{Ar})], 134.4[3 \mathrm{C},(\mathrm{Ar})], 164.3[6 \mathrm{C},(\underline{\mathrm{C}}=\mathrm{O})], 165.3[3 \mathrm{C},(\mathrm{C}=\mathrm{O})] \mathrm{ppm} ; \delta_{\mathrm{P}}\left\{{ }^{1} \mathrm{H}\right\}(202$ $\left.\mathrm{MHz}, \mathrm{CDCl}_{3}\right) 68.52 \mathrm{ppm}$; MALDI TOF MS calcd. for $\mathrm{C}_{63} \mathrm{H}_{87} \mathrm{O}_{21} \mathrm{PS}, \mathrm{M}=1242.5$. Found $m / z$ $=1282.5 \mathrm{M}+\mathrm{K}$.

Polyanionic dendrimer 16. Dendrimer 14 (FW 1243.4, $125 \mathrm{mg}, 0.1 \mathrm{mmol}$ ) was dissolved in dry dichloromethane $(1.5 \mathrm{~mL})$, and trifluoroacetic acid was added $(2 \mathrm{~mL})$. The reaction mixture was stirred at room temperature under argon atmosphere for $5 \mathrm{~h}$. All the volatiles were removed in vacuo, and the residue was dissolved in THF $(1 \mathrm{~mL})$. Next, acetone $(10 \mathrm{~mL})$ was added to that solution and the resulting mixture was kept in the refrigerator for about an hour. The precipitate was filtered off to provide acid $15(84 \mathrm{mg}, 93 \%)$ as a white solid. NMR: $\delta_{\mathrm{H}}\left(500 \mathrm{MHz}, \mathrm{CDCl}_{3}\right.$-THF-d $\mathrm{d}_{8}$ 1:5) 1.60-1.69 [m, 12H, $\left(\mathrm{POCH}_{2} \mathrm{CH}_{2} \mathrm{CH}_{2} \mathrm{CH}_{2} \mathrm{OC}\right)$ and $\left.\left(\mathrm{POCH}_{2} \mathrm{CH}_{2} \mathrm{CH}_{2} \mathrm{CH}_{2} \mathrm{OC}\right)\right], 3.86\left[\mathrm{dt},{ }^{3} \mathrm{~J}(\mathrm{H}, \mathrm{H})=6.0 \mathrm{~Hz},{ }^{3} J(\mathrm{P}, \mathrm{H})=12.6 \mathrm{~Hz}, 6 \mathrm{H}\right.$, 
$\left.\left(\mathrm{POCH}_{2} \mathrm{CH}_{2} \mathrm{CH}_{2} \mathrm{CH}_{2} \mathrm{OC}\right)\right], 4.11\left[\mathrm{t},{ }^{3} \mathrm{~J}(\mathrm{H}, \mathrm{H})=6.0 \mathrm{~Hz}, 6 \mathrm{H},\left(\mathrm{POCH}_{2} \mathrm{CH}_{2} \mathrm{CH}_{2} \mathrm{CH}_{2} \mathrm{OC}\right)\right.$ ], 8.52 [s, $6 \mathrm{H}, \mathrm{Ar}], 8.55$ [s, 3H, Ar] ppm; $\delta_{\mathrm{C}}\left(125 \mathrm{MHz}, \mathrm{CDCl}_{3}-\mathrm{THF}_{8} \mathrm{~d}_{8} 1: 5\right) 25.06,26.22\left[\mathrm{~d},{ }^{3} J(\mathrm{C}, \mathrm{P})=7.5\right.$ $\left.\mathrm{Hz}, 3 \mathrm{C},\left(\mathrm{OCH}_{2} \mathrm{CH}_{2} \mathrm{CH}_{2} \mathrm{CH}_{2} \mathrm{OP}\right)\right], 67.04\left[\mathrm{~d},{ }^{2} \mathrm{~J}(\mathrm{C}, \mathrm{P})=5.2 \mathrm{~Hz}, 3 \mathrm{C},\left(\mathrm{OCH}_{2} \mathrm{CH}_{2} \mathrm{CH}_{2} \mathrm{CH}_{2} \mathrm{OP}\right)\right]$, 67.25 [3C, $\left(\mathrm{OCH}_{2} \mathrm{CH}_{2} \mathrm{CH}_{2} \mathrm{CH}_{2} \mathrm{OP}\right)$ ], 130.6 [9C, (ipso Ar)], 131.3 [9C, (Ar)], 164.3 [3C, (C=O)], $165.6[6 \mathrm{C},(\underline{\mathrm{C}}=\mathrm{O})] \mathrm{ppm} ; \delta_{\mathrm{P}}\left\{{ }^{1} \mathrm{H}\right\}\left(202 \mathrm{MHz}, \mathrm{CDCl}_{3}-\mathrm{THF}_{8} \mathrm{~d}_{8} 1: 5\right) 68.52$ ppm; HRMS (TOF MS ES) $\mathrm{M}-\mathrm{H}^{+}$: found 905.1371. $\mathrm{C}_{39} \mathrm{H}_{39} \mathrm{O}_{21} \mathrm{PS}$ requires 905.1364. Soon after, acid 15 (35 mg, $0.0179 \mathrm{mmol}$ ) was dispersed in water $(2 \mathrm{~mL})$ and solid sodium bicarbonate (10 $\mathrm{mg}$, $0.11 \mathrm{mmol}, 6$ equiv.) was added. After $20 \mathrm{~min}$, the resulting solution was frozen and lyophilized under high vacuum $(0.1 \mathrm{mmHg})$ to give 16 as a hexasodium salt of 15 (40 $\mathrm{mg})$, as a nonhygroscopic white powder.

Second generation polyester thiophosphate dendrimer 17. Carboxy-terminated, first generation thiophosphate dendrimer 15 (FW 906.8, $40 \mathrm{mg}, 0.044 \mathrm{mmol}$ ) was dissolved in dry THF $(1.0 \mathrm{~mL})$. Then, the reaction mixture was diluted with dry dichloromethane $(2 \mathrm{~mL})$. Di-tert-butyl 5-hydroxymethylbenzene-1,3-dicarboxylate (2) (FW 308.4, $67 \mathrm{mg}, 0.3 \mathrm{mmol}$, 6.6 equiv.) and 1-ethyl-3-(3-dimethylaminopropyl)carbodiimide (EDC) $(70 \mathrm{mg}, 0.36 \mathrm{mmol}$, 8.0 equiv.) were added under argon atmosphere. The suspension was stirred vigorously and quickly dissolved. After $5 \mathrm{~min}$, 4-dimethylaminopyridine (DMAP) (FW 122.2, $5.0 \mathrm{mg}$, 0.04 mmol, 0.9 equiv.) was added and the mixture was stirred at room temperature overnight. The reaction mixture was then diluted with ethyl acetate $(20 \mathrm{~mL})$, washed with $0.1 \mathrm{M}$ citric acid $(10 \mathrm{~mL})$, dried $\left(\mathrm{MgSO}_{4}\right)$, and evaporated under reduced pressure. The residue was placed on a short plug of silica gel. Elution with the cyclohexane-acetone 100:1 mixture and gradually increasing the polarity to cyclohexane-acetone 15:1, afforded the title ester $17(95 \mathrm{mg})$. Yield $84 \% . \delta_{\mathrm{H}}\left(500 \mathrm{MHz}, \mathrm{CDCl}_{3}\right) 1.58\left[\mathrm{~s}, 108 \mathrm{H}, \mathrm{C}\left(\mathrm{CH}_{3}\right)_{3}\right], 1.80$ $1.87\left[\mathrm{~m}, 6 \mathrm{H},\left(\mathrm{POCH}_{2} \mathrm{CH}_{2} \mathrm{CH}_{2} \mathrm{CH}_{2} \mathrm{OC}\right)\right], 1.88-1.96\left[\mathrm{~m}, 6 \mathrm{H},\left(\mathrm{POCH}_{2} \mathrm{CH}_{2} \mathrm{CH}_{2} \mathrm{CH}_{2} \mathrm{OC}\right)\right], 4.12$ $\left[\mathrm{dt},{ }^{3} J(\mathrm{H}, \mathrm{H})=6.3 \mathrm{~Hz},{ }^{3} \mathrm{~J}(\mathrm{P}, \mathrm{H})=14.3 \mathrm{~Hz}, 6 \mathrm{H},\left(\mathrm{POCH}_{2} \mathrm{CH}_{2} \mathrm{CH}_{2} \mathrm{CH}_{2} \mathrm{OC}\right)\right], 4.40\left[\mathrm{t},{ }^{3} J(\mathrm{H}, \mathrm{H})\right.$ $\left.=6.4 \mathrm{~Hz}, 6 \mathrm{H},\left(\mathrm{POCH}_{2} \mathrm{CH}_{2} \mathrm{CH}_{2} \mathrm{CH}_{2} \mathrm{OC}\right)\right], 5.46\left[\mathrm{~s}, 12 \mathrm{H},\left(\mathrm{ArCH}_{2}\right)\right], 8.20\left(\mathrm{~s}, 12 \mathrm{H}, \mathrm{Ar}^{2}\right), 8.53$ $\left(\mathrm{s}, 6 \mathrm{H}, \mathrm{Ar}^{2}\right), 8.85\left(\mathrm{~s}, 3 \mathrm{H}, \mathrm{Ar}^{1}\right), 8.87\left(\mathrm{~s}, 6 \mathrm{H}, \mathrm{Ar}^{1}\right) \mathrm{ppm} ; \delta_{\mathrm{C}}\left(125 \mathrm{MHz}, \mathrm{CDCl}_{3}\right) 25.01$ [3C, $\left.\left.\mathrm{O}=\mathrm{COCH}_{2} \underline{\mathrm{CH}}_{2} \mathrm{CH}_{2} \mathrm{CH}_{2} \mathrm{O}\right)\right], 26.66\left[\mathrm{~d},{ }^{3} \mathrm{~J}(\mathrm{C}, \mathrm{P})=7.6 \mathrm{~Hz}, 3 \mathrm{C},\left(\mathrm{OCH}_{2} \mathrm{CH}_{2} \mathrm{CH}_{2} \mathrm{CH}_{2} \mathrm{OP}\right)\right], 28.16$ [36C, $\left.\mathrm{C}\left(\mathrm{CH}_{3}\right)_{3}\right], 65.13$ [3C, $\left.\left(\mathrm{OCH}_{2} \mathrm{CH}_{2} \mathrm{CH}_{2} \mathrm{CH}_{2} \mathrm{OP}\right)\right], 66.51\left[6 \mathrm{C},\left(\mathrm{ArCH} \underline{H}_{2}\right)\right], 67.61\left[\mathrm{~d},{ }^{2} J(\mathrm{C}, \mathrm{P})\right.$ $\left.=5.7 \mathrm{~Hz}, 3 \mathrm{C},\left(\mathrm{OCH}_{2} \mathrm{CH}_{2} \mathrm{CH}_{2} \underline{\mathrm{CH}}_{2} \mathrm{OP}\right)\right], 81.87$ [12C, $\left.\mathrm{C}\left(\mathrm{CH}_{3}\right)_{3}\right], 130.6$ [6C, $\left.\left(\mathrm{Ar}^{2}\right)\right], 130.9$ [6C, (ipso $\left.\mathrm{Ar}^{1}\right)$ ], 131.6 [3C, (ipso $\left.\mathrm{Ar}^{1}\right)$ ], 132.8 [12C, (ipso $\left.\mathrm{Ar}^{2}\right)$ ], 133.1 [12C, $\left(\mathrm{Ar}^{2}\right)$ ], 134.9 [3C, $\left(\mathrm{Ar}^{1}\right)$ ], 135.0 [6C, $\left.\left(\mathrm{Ar}^{1}\right)\right], 136.0\left[6 \mathrm{C},\left(\right.\right.$ ipso $\left.\left.\mathrm{Ar}^{2}\right)\right], 164.6[21 \mathrm{C},(\mathrm{C}=\mathrm{O})] \mathrm{ppm} ; \delta_{\mathrm{P}}\left\{{ }^{1} \mathrm{H}\right\}\left(202 \mathrm{MHz}, \mathrm{CD}_{3} \mathrm{OD}-\right.$ $\mathrm{CDCl}_{3}$ 2:1) 68.60 ppm; MALDI TOF MS calcd. for $\mathrm{C}_{141} \mathrm{H}_{171} \mathrm{O}_{45} \mathrm{PS}, \mathrm{M}=2647.0$. Found $\mathrm{m} / \mathrm{z}$ $=2670.4 \mathrm{M}+\mathrm{Na}, 2686.7 \mathrm{M}+\mathrm{K}$.

Polyanionic dendrimer 19. Dendrimer 17 (FW 2648.9, $70 \mathrm{mg}, 0.0264 \mathrm{mmol}$ ) was dissolved in dry dichloromethane $(1.5 \mathrm{~mL})$, and trifluoroacetic acid was added $(1.5 \mathrm{~mL})$. The reaction mixture was stirred at room temperature under argon atmosphere for $5 \mathrm{~h}$. All the volatiles were removed in vacuo, and the residue was dissolved in THF $(1 \mathrm{~mL})$. Next, acetone $(10 \mathrm{~mL})$ was added to that solution and the resulting mixture was kept in the refrigerator for about an hour. The precipitate was filtered off to provide acid 18 (48 $\mathrm{mg}, 92 \%)$ as a white solid. NMR: $\delta_{\mathrm{H}}\left(500 \mathrm{MHz}, \mathrm{THF}-\mathrm{d}_{8}\right) 1.82-1.88$ [m, 6H, $\left.\left(\mathrm{POCH}_{2} \mathrm{CH}_{2} \mathrm{CH}_{2} \mathrm{CH}_{2} \mathrm{OC}\right)\right], 1.90-1.98[\mathrm{~m}, 6 \mathrm{H}$, $\left.\left(\mathrm{POCH}_{2} \mathrm{CH}_{2} \mathrm{CH}_{2} \mathrm{CH}_{2} \mathrm{OC}\right)\right], 3.59-3.64\left[\mathrm{~m}, 6 \mathrm{H},\left(\mathrm{POCH}_{2} \mathrm{CH}_{2} \mathrm{CH}_{2} \mathrm{CH}_{2} \mathrm{OC}\right)\right], 3.80\left[\mathrm{t},{ }^{3} \mathrm{~J}(\mathrm{H}, \mathrm{H})=\right.$ $\left.6.8 \mathrm{~Hz}, 6 \mathrm{H},\left(\mathrm{POCH}_{2} \mathrm{CH}_{2} \mathrm{CH}_{2} \mathrm{CH}_{2} \mathrm{OC}\right)\right], 5.47$ [s, 12H, $\left.\left(\mathrm{ArCH}_{2}\right)\right], 8.30\left(\mathrm{~s}, 12 \mathrm{H}, \mathrm{Ar}^{2}\right), 8.36(\mathrm{~s}, 6 \mathrm{H}$, $\left.\mathrm{Ar}^{2}\right), 8.54\left(\mathrm{~s}, 6 \mathrm{H}, \mathrm{Ar}^{1}\right), 8.61\left(\mathrm{~s}, 3 \mathrm{H}, \mathrm{Ar}^{1}\right), 10.87$ (brs, 12H, COOH) ppm; $\delta_{\mathrm{C}}(125 \mathrm{MHz}, \mathrm{THF}-$ $\left.\mathrm{d}_{8}\right) 25.40\left[3 \mathrm{C}, \mathrm{O}=\mathrm{COCH}_{2} \mathrm{CH}_{2} \mathrm{CH}_{2} \mathrm{CH}_{2} \mathrm{O}\right)$ ], 28.96 [3C, $\left.\left(\mathrm{OCH}_{2} \mathrm{CH}_{2} \mathrm{CH}_{2} \mathrm{CH}_{2} \mathrm{OP}\right)\right], 65.92$ [3C, $\left.\left(\mathrm{OCH}_{2} \mathrm{CH}_{2} \mathrm{CH}_{2} \mathrm{CH}_{2} \mathrm{OP}\right)\right], 66.96$, [3C, $\left.\left(\mathrm{OCH}_{2} \mathrm{CH}_{2} \mathrm{CH}_{2} \mathrm{CH}_{2} \mathrm{OP}\right)\right], 67,24$ [6C, $\left.\left(\mathrm{ArCH}_{2}\right)\right], 128.6$ [9C, $\left.\left(\mathrm{Ar}^{1}\right)\right], 130.5$ [12C, (ipso $\left.\left.\mathrm{Ar}^{2}\right)\right], 130.9$ [6C, $\left(\right.$ ipso $\left.\left.\mathrm{Ar}^{2}\right)\right], 131.6\left[6 \mathrm{C},\left(\mathrm{Ar}^{2}\right)\right], 133.5$ [12C, $\left.\left(\mathrm{Ar}^{2}\right)\right]$, 137.1 [9C, (ipso Ar $\left.{ }^{1}\right)$ ], 163.9 [3C, $\left.(\underline{C}=\mathrm{O})\right], 164.5$ [6C, $\left.(\underline{C}=\mathrm{O})\right], 165.8$ [12C, $\left.(\mathrm{HOC}=\mathrm{O})\right]$ ppm $\delta_{\mathrm{P}}$ $\left\{{ }^{1} \mathrm{H}\right\}\left(202 \mathrm{MHz}, \mathrm{THF}-\mathrm{d}_{8}\right) 68.71$ ppm; MALDI TOF MS calcd. for $\mathrm{C}_{93} \mathrm{H}_{75} \mathrm{O}_{45} \mathrm{PS}, \mathrm{M}=1974.3$. Found $m / z$, fragmentation: 1149.8, 1121.7, 1093.5, 1065.5 (major peaks). Elemental analysis: Found (acid): C, 56.51; H, 3.85; S, 1.62\%. $\mathrm{C}_{93} \mathrm{H}_{75} \mathrm{O}_{45} \mathrm{PS}$ requires $\mathrm{C}, 56.54 ; \mathrm{H}, 3.83 ; \mathrm{S}, 1.62 \%$. Afterwards, acid 18 (35 mg, $0.0179 \mathrm{mmol})$ was dispersed in water $(2 \mathrm{~mL})$ and solid sodium bicarbonate (18 mg, $0.2 \mathrm{mmol}, 12$ equiv.) was added. After $20 \mathrm{~min}$, the resulting solution 
was frozen and lyophilized under high vacuum $(0.1 \mathrm{mmHg})$ to give 19 as a dodecasodium salt of 18 (40 mg), as a nonhygroscopic white powder.

Second generation fully protected dendrimer 21. Hydroxy-terminated, first generation thiophosphate dendrimer ${ }^{12} 20$ (FW 1009.1, $51 \mathrm{mg}, 0.050 \mathrm{mmol}$ ) was suspended in dry dichloromethane (3.0 mL). Di-tert-butyl 1.3.5-benzenetricarboxylate (1) (FW 322.4, $105 \mathrm{mg}, 0.325 \mathrm{mmol}$, 6.5 equiv.) and 1-ethyl-3-(3-dimethylaminopropyl)carbodiimide (EDC) $(77 \mathrm{mg}, 0.4 \mathrm{mmol}$, 8.0 equiv.) were added under argon atmosphere. The suspension was stirred vigorously and quickly dissolved. After 5 min, 4-dimethylaminopyridine (DMAP) (FW 122.2, $5.0 \mathrm{mg}, 0.04 \mathrm{mmol}, 0.9$ equiv.) was added and the mixture was stirred at room temperature overnight. The reaction mixture was then diluted with ethyl acetate $(20 \mathrm{~mL})$, washed with $0.1 \mathrm{M}$ citric acid $(10 \mathrm{~mL})$, dried $\left(\mathrm{MgSO}_{4}\right)$, and evaporated under reduced pressure. The residue was placed on a short plug of silica gel. Elution with the neat dichloromethane provided the title dendrimer $21(127 \mathrm{mg})$. Yield $90 \% . \delta_{\mathrm{H}}(500 \mathrm{MHz}$, $\left.\mathrm{CDCl}_{3}\right) 1.58$ [s, $\left.108 \mathrm{H}, \mathrm{C}\left(\mathrm{CH}_{3}\right)_{3}\right], 1.78-1.87$ [m, 12H, $\left.\left(\mathrm{POCH}_{2} \mathrm{CH}_{2} \mathrm{CH}_{2} \mathrm{CH}_{2} \mathrm{OC}\right)\right], 1.88-1.95$ [m, 12H, $\left.\left(\mathrm{POCH}_{2} \mathrm{CH}_{2} \mathrm{CH}_{2} \mathrm{CH}_{2} \mathrm{OC}\right)\right], 2.12-2.21\left[\mathrm{~m}, 6 \mathrm{H},\left(\mathrm{POCH}_{2} \mathrm{CH}_{2} \mathrm{CH}_{2} \mathrm{OP}\right)\right], 4.12$ [dt, $\left.{ }^{3} J(\mathrm{H}, \mathrm{H})=6.3 \mathrm{~Hz},{ }^{3} J(\mathrm{P}, \mathrm{H})=13.6 \mathrm{~Hz}, 12 \mathrm{H},\left(\mathrm{POCH}_{2} \mathrm{CH}_{2} \mathrm{CH}_{2} \mathrm{CH}_{2} \mathrm{OC}\right)\right], 4.24\left[\mathrm{dt},{ }^{3} \mathrm{~J}(\mathrm{H}, \mathrm{H})\right.$ $\left.=6.3 \mathrm{~Hz},{ }^{3} \mathrm{~J}(\mathrm{P}, \mathrm{H})=14.3 \mathrm{~Hz}, 12 \mathrm{H},\left(\mathrm{POC}_{\underline{\mathrm{H}}} \mathrm{CH}_{2} \mathrm{CH}_{2} \mathrm{OP}\right)\right], 4.45\left[\mathrm{t},{ }^{3} \mathrm{~J}(\mathrm{H}, \mathrm{H})=6.1 \mathrm{~Hz}, 12 \mathrm{H}\right.$, $\left.\left(\mathrm{POCH}_{2} \mathrm{CH}_{2} \mathrm{CH}_{2} \mathrm{CH}_{2} \mathrm{OC}\right)\right], 8.70(\mathrm{~s}, 6 \mathrm{H}, \mathrm{Ar}), 8.71(\mathrm{~s}, 12 \mathrm{H}, \mathrm{Ar}) \mathrm{ppm} ; \delta_{\mathrm{C}}\left(125 \mathrm{MHz}, \mathrm{CDCl}_{3}\right)$, 25.07 [6C, O=COCH $\left.\left.{ }_{2} \mathrm{CH}_{2} \mathrm{CH}_{2} \mathrm{CH}_{2} \mathrm{O}\right)\right], 26.71\left[\mathrm{~d},{ }^{3} \mathrm{~J}(\mathrm{C}, \mathrm{P})=7.8 \mathrm{~Hz}, 6 \mathrm{C},\left(\mathrm{OCH}_{2} \mathrm{CH}_{2} \mathrm{CH}_{2} \mathrm{CH}_{2} \mathrm{OP}\right)\right]$, 28.13 [36C, $\left.\mathrm{C}\left(\mathrm{CH}_{3}\right)_{3}\right], 29.41-29.49\left[\mathrm{~m},{ }^{3} \mathrm{~J}(\mathrm{C}, \mathrm{P})=7.8 \mathrm{~Hz}, 3 \mathrm{C}\right.$, $\left.\left(\mathrm{POCH}_{2} \mathrm{CH}_{2} \mathrm{CH}_{2} \mathrm{OP}\right)\right], 61.62-$ $61.73\left[\mathrm{~m}, 6 \mathrm{C},\left(\mathrm{POCH}_{2} \mathrm{CH}_{2} \mathrm{CH}_{2} \mathrm{OP}\right)\right], 64.87$ [6C, $\left.\left(\mathrm{OCCH}_{2} \mathrm{CH}_{2} \mathrm{CH}_{2} \mathrm{CH}_{2} \mathrm{OP}\right)\right], 67.64\left[\mathrm{~d},{ }^{2} \mathrm{~J}(\mathrm{C}, \mathrm{P})\right.$ $\left.=5.4 \mathrm{~Hz}, 6 \mathrm{C},\left(\mathrm{OCH}_{2} \mathrm{CH}_{2} \mathrm{CH}_{2} \mathrm{CH}_{2} \mathrm{OP}\right)\right], 82.17$ [12C, $\mathrm{C}\left(\mathrm{CH}_{3}\right)_{3}$ ], 130.9 [6C, (ipso Ar)], 132.8 [12C, (ipso Ar)], 134.0 [12C, $(\mathrm{Ar})], 134.4$ [6C, $(\mathrm{Ar})], 164.2$ [12C, $(\underline{\mathrm{C}}=\mathrm{O})], 165.2$ [6C, $(\underline{\mathrm{C}}=\mathrm{O})]$ ppm; $\delta_{\mathrm{P}}\left\{{ }^{1} \mathrm{H}\right\}\left(202 \mathrm{MHz}, \mathrm{CDCl}_{3}\right), 68.29\left(\mathrm{P}^{0}\right), 68.58\left(3 \mathrm{P}^{1}\right) \mathrm{ppm}$; MALDI TOF MS calcd. for $\mathrm{C}_{135} \mathrm{H}_{192} \mathrm{O}_{48} \mathrm{P}_{4} \mathrm{~S}_{4}, \mathrm{M}=2833$.0. Found $m / z=2845.8 \mathrm{M}+\mathrm{Na}$.

Polyanionic dendrimer 23. Dendrimer $21(80 \mathrm{mg}, 0.028 \mathrm{mmol})$ was dissolved in dry dichloromethane $(1.5 \mathrm{~mL})$, and trifluoroacetic acid was added $(1.5 \mathrm{~mL})$. The reaction mixture was stirred at room temperature under argon atmosphere for $5 \mathrm{~h}$. All the volatiles were removed in vacuo, and the residue was dissolved in THF $(1 \mathrm{~mL})$. Next, acetone $(11 \mathrm{~mL})$ was added to that solution and the resulting mixture was kept in the refrigerator for about an hour. The precipitate was filtered off to provide acid $22(56 \mathrm{mg}, 93 \%)$ as a white solid. NMR: $\delta_{\mathrm{H}}\left(200 \mathrm{MHz}, \mathrm{CD}_{3} \mathrm{OD}\right)$ 1.75-1.96 [m, 24H, $\left.\left(\mathrm{POCH}_{2} \mathrm{CH}_{2} \mathrm{C}_{2} \mathrm{CH}_{2} \mathrm{OC}\right)\right], 2.12-2.21[\mathrm{~m}, 6 \mathrm{H}$, $\left.\left(\mathrm{POCH}_{2} \mathrm{CH}_{2} \mathrm{CH}_{2} \mathrm{OP}\right)\right], 4.09-4.21\left[\mathrm{~m},{ }^{3} \mathrm{~J}(\mathrm{H}, \mathrm{H})=6.3 \mathrm{~Hz}, 12 \mathrm{H},\left(\mathrm{POCH}_{2} \mathrm{CH}_{2} \mathrm{CH}_{2} \mathrm{CH}_{2} \mathrm{OC}\right)\right]$, 4.24$4.30\left[\mathrm{~m}, 12 \mathrm{H},\left(\mathrm{POCH}_{2} \mathrm{CH}_{2} \mathrm{CH}_{2} \mathrm{OP}\right)\right], 4.37\left[\mathrm{t},{ }^{3} \mathrm{~J}(\mathrm{H}, \mathrm{H})=6.0 \mathrm{~Hz}, 12 \mathrm{H},\left(\mathrm{POCH}_{2} \mathrm{CH}_{2} \mathrm{CH}_{2} \mathrm{CH}_{2} \mathrm{OC}\right)\right]$, $8.70(\mathrm{~s}, 12 \mathrm{H}, \mathrm{Ar}), 8.74(\mathrm{~s}, 6 \mathrm{H}, \mathrm{Ar}) \mathrm{ppm} ; \delta_{\mathrm{C}}\left(125 \mathrm{MHz}, \mathrm{CD}_{3} \mathrm{OD}-\mathrm{CDCl}_{3} 3: 1\right), 26.44$ [6C, $\left.\left.\mathrm{O}=\mathrm{COCH}_{2} \mathrm{CH}_{2} \mathrm{CH}_{2} \mathrm{CH}_{2} \mathrm{O}\right)\right], 28.09\left[\mathrm{~d},{ }^{3} J(\mathrm{C}, \mathrm{P})=7.4 \mathrm{~Hz}, 6 \mathrm{C},\left(\mathrm{OCH}_{2} \mathrm{CH}_{2} \mathrm{CH}_{2} \mathrm{CH}_{2} \mathrm{OP}\right)\right], 30.60-$ $30.70\left[\mathrm{~m}, 3 \mathrm{C},\left(\mathrm{POCH}_{2} \mathrm{CH}_{2} \mathrm{CH}_{2} \mathrm{OP}\right)\right], 66.27-66.40\left[\mathrm{~m}, 6 \mathrm{C},\left(\mathrm{POCH}_{2} \mathrm{CH}_{2} \mathrm{CH}_{2} \mathrm{OP}\right)\right], 66.53$ [6C, $\left.\left(\mathrm{OCH}_{2} \mathrm{CH}_{2} \mathrm{CH}_{2} \mathrm{CH}_{2} \mathrm{OP}\right)\right], 69.15\left[\mathrm{~d},{ }^{2} \mathrm{~J}(\mathrm{C}, \mathrm{P})=5.6 \mathrm{~Hz}, 6 \mathrm{C},\left(\mathrm{OCH}_{2} \mathrm{CH}_{2} \mathrm{CH}_{2} \mathrm{CH}_{2} \mathrm{OP}\right)\right], 132.5$ [6C, (ipso Ar)], 133.3 [6C, (Ar)], 135.6 [12C, (Ar)], 136.1 [12C, (ipso Ar)], 166.6 [6C, (C=O)], 167.9 [12C, $(\mathrm{C}=\mathrm{O})] \mathrm{ppm} ; \delta_{\mathrm{P}}\left\{{ }^{1} \mathrm{H}\right\}\left(202 \mathrm{MHz}, \mathrm{CD}_{3} \mathrm{OD}-\mathrm{CDCl}_{3} 3: 1\right), 68.61\left(\mathrm{P}^{0}\right), 68.80\left(3 \mathrm{P}^{1}\right)$ ppm; MALDI TOF MS calcd. for $\mathrm{C}_{87} \mathrm{H}_{96} \mathrm{O}_{48} \mathrm{P}_{4} \mathrm{~S}_{4}, \mathrm{M}=2160.3$. Found $m / z$, fragmentation: 1470.0, 1450.8, 1005.7, 947.5, 930.7 (major peaks). Elemental analysis: Found (acid): C, $48.39 ; \mathrm{H}, 4.41 ; \mathrm{S}, 6.00 \%$. $\mathrm{C}_{87} \mathrm{H}_{96} \mathrm{O}_{48} \mathrm{P}_{4} \mathrm{~S}_{4}$ requires $\mathrm{C}, 48.34 ; \mathrm{H}, 4.48 ; \mathrm{S}, 5.93 \%$. Finally, acid $22(40 \mathrm{mg}, 0.0185 \mathrm{mmol})$ was dispersed in water $(2 \mathrm{~mL})$ and solid sodium bicarbonate (19 $\mathrm{mg}, 0.22 \mathrm{mmol}, 12$ equiv.) was added. After $20 \mathrm{~min}$, the resulting solution was frozen and lyophilized under high vacuum $(0.1 \mathrm{~mm} \mathrm{Hg})$ to give $\mathbf{2 3}$ as a dodecasodium salt of 22 $(47 \mathrm{mg})$, as a nonhygroscopic white powder.

\section{Conclusions}

In conclusion, using simple and readily available monomers, an easy and highly efficient method for the synthesis of new polyanionic dendrimers as potential broadspectrum antiviral drugs in their own right was presented. The mild conditions of both the coupling and virtually quantitative deprotection reactions, provided highly pure and watersoluble macromolecular materials in good overall yields. For example, the total yields 
of the key compounds were as follows: Second generation acid 8-64\%, third generation polyacid $11-42 \%$, and second generation acid $18-60 \%$. The roughly estimated time of the preparation of each generation (two steps-condensation, purification, cleavage of the terminal esters, and purification) should fit easily within the 2-day-period. The important advantage of the presented strategy is direct access to the polyanionic material at each generation of the prepared dendrimer. Therefore, the synthesis is at least one difficult synthetic step shorter. This approach seems to be somehow a general methodology, which enables the transformation of practically any macromolecular compound terminated with hydroxy functions, into its polyanionic derivative. Moreover, it offers the possibility to make discrete modifications layer by layer (i.e., $\mathrm{P}=\mathrm{O}, \mathrm{P}=\mathrm{S}$, and/or carbon branching) within the same dendrimer skeleton, a key for a structure-activity relationship study.

Although the chemical synthesis of dendrimers is more than two decades old, the most significant reason hampering the broader use of dendrimers in biomedicine is usually difficult and their multistep preparation is time-consuming, especially for the high generation structures. Consequently, developing methodologies offering faster access to the important macromolecular material is especially warranted.

Supplementary Materials: ${ }^{1} \mathrm{H},{ }^{31} \mathrm{P},{ }^{13} \mathrm{C}$ NMR, and mass spectra of the products synthesized in this work are available online.

Funding: This research received no external funding.

Institutional Review Board Statement: Not applicable.

Informed Consent Statement: Not applicable.

Data Availability Statement: The data presented in this study are available in the Supplementary Materials.

Acknowledgments: The author thanks The Polish Academy of Sciences for financial support.

Conflicts of Interest: The author declares no conflict of interest.

Sample Availability: Samples of the polyanionic dendrimers 6, 9, 12, 16, 19, 23 are available from the author.

\section{References}

1. Contin, M.; Garcia, C.; Dobrecky, C.; Lucangioli, S.; D'Accorso, N. Advances in drug delivery, gene delivery and therapeutic agents based on dendritic materials. Future Med. Chem. 2019, 11, 1791-1810. [CrossRef] [PubMed]

2. Gurunathan, S.; Qasim, M.; Choi, Y.; Tae Do, J.; Park, C.; Hong, K.; Kim, J.-H.; Song, H. Antiviral Potential of Nanoparticles-Can Nanoparticles Fight Against Coronaviruses? Nanomaterials 2020, 10, 1645. [CrossRef] [PubMed]

3. Ortega, M.Á.; Guzmán Merino, A.; Fraile-Martínez, O.; Recio-Ruiz, J.; Pekarek, L.; Guijarro, L.G.; García-Honduvilla, N.; Álvarez-Mon, M.; Buján, J.; García-Gallego, S. Dendrimers and Dendritic Materials: From Laboratory to Medical Practice in Infectious Diseases. Pharmaceutics 2020, 12, 874. [CrossRef] [PubMed]

4. McCarthy, T.D.; Karellas, P.; Henderson, S.A.; Giannis, M.; O'Keefe, D.F.; Heery, G.; Paull, J.R.A.; Matthews, B.R.; Holan, G. Dendrimers as Drugs: Discovery and Preclinical and Clinical Development of Dendrimer-Based Microbicides for HIV and STI Prevention. Mol. Pharmacol. 2005, 2, 312-318. [CrossRef] [PubMed]

5. Rojo, J.; Delgado, R. Dendrimers and Dendritic Polymers as Anti-infective Agents: New Antimicrobial Strategies for Therapeutic Drugs. Anti-Infect. Agents 2007, 6, 151-174. [CrossRef]

6. Jimenez, J.L.; Pion, M.; de la Mata, F.J.; Gomez, R.; Muñoz, E.; Leal, M.; Muñoz-Fernández, M.A. Dendrimers as topical microbicides with activity against HIV. New J. Chem. 2012, 36, 299-309. [CrossRef]

7. Sepúlveda-Crespo, D.; Lorente, R.; Leal, M.; Gomez, R.; de la Mata, F.J.; Jimenez, J.L.; Muñoz-Fernández, M.A. Synergistic activity profile of carbosilane dendrimer G2-STE16 in combination with other dendrimers and antiretrovirals as topical anti-HIV-1 microbicide. Nanomedicine 2014, 10, 609-618. [CrossRef]

8. García-Gallego, S.; Díaz, L.; Jimenez, J.L.; Gomez, R.; de la Mata, F.J.; Muñoz-Fernández, M.A. HIV-1 antiviral behavior of anionic PPI metallo-dendrimers with EDA core. Eur. J. Med. Chem. 2015, 98, 139-148. [CrossRef] [PubMed]

9. Galán, M.; Sánchez Rodríguez, J.; Jiménez, J.L.; Relloso, M.; Maly, M.; de la Mata, FJ.; Muñoz-Fernández, M.A.; Gómez, R. Synthesis of new anionic carbosilane dendrimers via thiol-ene chemistry and their antiviral behavior. Org. Biomol. Chem. 2014, 12, 3222-3227. [CrossRef] [PubMed]

10. Lüscher-Mattli, M. Polyanions-a lost chance in the fight against HIV and other virus diseases? Antivir. Chem. Chemother. 2000, 11, 249-259. [CrossRef] [PubMed] 
11. Cutler, B.; Justman, J. Vaginal microbicides and the prevention of HIV transmission. Lancet Infect. Dis. 2008, 8, 685-697. [CrossRef]

12. Whaley, K.J.; Hanes, J.; Shattock, R.; Cone, R.A.; Friend, D.R. Novel Approaches to Vaginal Delivery and Safety of Microbicides: Biopharmaceuticals, Nanoparticles, and Vaccines. Antivir. Res. 2010, 88, S55-S66. [CrossRef]

13. Telwatte, S.; Moore, K.; Johnson, A.; Tyssen, D.; Sterjovski, J.; Aldunate, M.; Gorry, P.R.; Ramsland, P.A.; Lewis, G.R.; Paull, J.R.A.; et al. Virucidal Activity of the Dendrimer Microbicide SPL7013 Against HIV-1. Antivir. Res. 2011, 90, 195-199. [CrossRef] [PubMed]

14. Ham, A.S.; Rohan, L.C.; Boczar, A.; Yang, L.; Buckheit, K.W.; Buckheit, R.W. Vaginal film drug delivery of the pyrimidinedione IQP-0528 for the prevention of HIV infection. Pharm. Res. 2012, 29, 1897-1907. [CrossRef] [PubMed]

15. Malik, N.; Wiwattanapatapee, R.; Klopsch, R.; Lorenz, K.; Frey, H.; Weener, J.W.; Meijer, E.W.; Paulus, W.; Duncan, R. Dendrimers: Relationship between structure and biocompatibility in vitro, and preliminary studies on the biodistribution of ${ }^{125} \mathrm{I}$-labelled polyamidoamine dendrimers in vivo. J. Control. Release 2000, 65, 133-148. [CrossRef]

16. Jevprasesphant, R.; Penny, J.; Jalal, R.; Attwood, D.; McKownand, N.B.; D’Emmanuele, A. The influence of surface modification on the cytotoxicity of PAMAM dendrimers. Int. J. Pharm. 2003, 252, 263-266. [CrossRef]

17. Starpharma Holdings Limited Website. Available online: https:/ / www.starpharma.com/vivagel (accessed on 24 June 2021).

18. Starpharma Holdings Limited Website. Available online: https://starpharma.com/vivagel/vivagel_clinical_trials (accessed on 28 May 2021).

19. Starpharma Holdings Limited Announcement on 15 April 2020. Available online: https://starpharma.com/news-room (accessed on 29 April 2021).

20. Starpharma Holdings Limited Website. Available online: https://starpharma.com/vivagel/spl7013-covid-19-nasal-spray (accessed on 24 June 2021).

21. Salamończyk, G.M.; Kuźnikowski, M.; Skowrońska, A. A divergent synthesis of thiophosphate-based dendrimers. Tetrahedron Lett. 2000, 41, 1643-1645. [CrossRef]

22. Salamończyk, G.M.; Kuźnikowski, M.; Poniatowska, E. Synthesis and oxygenation of selenophosphate dendrimers. Chem. Commun. 2001, 2202-2203. [CrossRef]

23. Salamończyk, G.M.; Kuźnikowski, M.; Poniatowska, E. Dendrimers bearing three types of branching functions. Tetrahedron Lett. 2002, 43, 1747-1749. [CrossRef]

24. Poniatowska, E.; Salamończyk, G.M. Phosphite dendrimers and their organometallic derivatives. Tetrahedron Lett. 2003, 44, 4315-4317. [CrossRef]

25. Salamończyk, G.M. Acyclovir terminated thiophosphate dendrimers. Tetrahedron Lett. 2003, 44, 7449-7453. [CrossRef]

26. Salamończyk, G.M. Synthesis of new dendrimers-Trimesic acid derivatives. Tetrahedron Lett. 2011, 52, 155-158. [CrossRef]

27. Salamończyk, G.M. Efficient synthesis of water-soluble, phosphonate-terminated polyester dendrimers. Tetrahedron Lett. 2015, 56, 7161-7164. [CrossRef]

28. Salamończyk, G.M. New water-soluble polyanionic dendrimers-Phosphoric and 1,3,5-benzenetricarboxylic acid derivatives. Tetrahedron 2012, 68, 10209-10217. [CrossRef]

29. Salamończyk, G.M.; Claes, S.; Oeyen, M.; Schols, D. Strong Antiviral Effect of Third Generation Polyanionic, Heterorganic Dendrimers Against HIV; The Polish Academy of Sciences: Sienkiewicza, Poland, 2021; Manuscript in Preparation.

30. Gao, W.-Y.; Sur, A.; Wang, C.-H.; Lorzing, G.R.; Antonio, A.M.; Taggart, G.A.; Ezazi, A.A.; Bhuvanesh, N.E.; Bloch, D.; Powers, D.C. Atomically Precise Crystalline Materials Based on Kinetically Inert Metal Ions via Reticular Mechanopolymerization. Angew. Chem. Int. Ed. 2020, 59, 10878-10883. [CrossRef] [PubMed]

31. Matteucci, M.D.; Caruthers, M.H. Synthesis of deoxyoligonucleotides on a polymer support. J. Am. Chem. Soc. 1981, 103, 3185-3191. [CrossRef]

32. Beaucage, S.L.; Iyer, R.P. Advances in the Synthesis of Oligonucleotides by the Phosphoramidite Approach. Tetrahedron 1992, 48, 2223-2311. [CrossRef]

33. Salamończyk, G.M.; Pietrusiewicz, K.M. Expedient synthesis of D-myo-inositol 1,4,5-trisphosphate and D-myo-inositol 1,4bisphosphate. Tetrahedron Lett. 1991, 32, 6167-6170. [CrossRef] 